TRANSACTIONS OF THE

AMERICAN MATHEMATICAL SOCIETY

Volume 359, Number 10, October 2007, Pages 4945-4975

S 0002-9947(07)04159-1

Article electronically published on May 16, 2007

\title{
HOMOLOGICAL INTEGRAL OF HOPF ALGEBRAS
}

\author{
D.-M. LU, Q.-S. WU, AND J. J. ZHANG
}

\begin{abstract}
The left and right homological integrals are introduced for a large class of infinite dimensional Hopf algebras. Using the homological integrals we prove a version of Maschke's theorem for infinite dimensional Hopf algebras. The generalization of Maschke's theorem and homological integrals are the keys to studying noetherian regular Hopf algebras of Gelfand-Kirillov dimension one.
\end{abstract}

\section{INTRODUCTION}

Let $H$ be a Hopf algebra over a base field $k$. A result of Larson and Radford [LR1, LR2] states that if $H$ is finite dimensional and if char $k=0$, then the following two conditions are equivalent:

(GLD) $H$ has global dimension 0, namely, $H$ is semisimple artinian.

(ANT) $S^{2}=\operatorname{id}_{H}$ where $S$ is the antipode of $H$.

A result of Larson and Sweedler [LS] states that (GLD) is equivalent to:

(ITG) $\epsilon\left(\int^{r}\right) \neq 0$ where $\int^{r}$ is the space of right integrals of $H$.

Larson and Sweedler's result (GLD) $\Leftrightarrow$ (ITG) is a generalization of Maschke's theorem for finite groups. These results are so elegant and useful that we cannot help attempting to extend them to the infinite dimensional case. The extension of $(\mathrm{ANT}) \Rightarrow(\mathrm{GLD})$ is quite successful. In WZ2, 0.1] the authors proved the following: Suppose that $H$ is a finite module over its affine center and that char $k=0$. If $S^{2}=\mathrm{id}_{H}$, then $H$ has finite global dimension. It is well-known that the converse of WZ2, 0.1] is false [Example 2.7. Until now the extension of (GLD) $\Leftrightarrow$ (ITG) has been less successful, partly due to the fact that we don't have a good definition of the left and right integrals of an infinite dimensional Hopf algebra. In this paper we use homological properties to define the integrals for a large class of infinite dimensional Hopf algebras, and then generalize the result of Larson and Sweedler $(\mathrm{GLD}) \Leftrightarrow(\mathrm{ITG})$.

To define the homological integral we need to assume that $H$ is Artin-Schelter Gorenstein, which is satisfied by many noetherian Hopf algebras [Section 1]. The left homological integral $\int^{l}$ of $H$ is the 1-dimensional $H$-bimodule $\operatorname{Ext}_{H}^{d}\left({ }_{H} k,{ }_{H} H\right)$ where $d$ is the injective dimension of $H$. The right homological integral $\int^{r}$ is defined similarly [Definition 1.1. In the finite dimensional case the left and right homological integrals agree with the usual left and right integrals of $H$ respectively.

Received by the editors May 16, 2005 and, in revised form, July 11, 2005.

2000 Mathematics Subject Classification. Primary 16A62, 16W30; Secondary 16E70, 20J50.

Key words and phrases. Hopf algebra, homological integral, Gorenstein property, regularity, Gelfand-Kirillov dimension, integral order, integral quotient, PI degree.

(C)2007 American Mathematical Society Reverts to public domain 28 years from publication 
We say $H$ satisfies (Cond1) if the map

$$
\operatorname{Ext}_{H}^{d}\left(\int^{r}, \epsilon\right): \quad \operatorname{Ext}_{H}^{d}\left(\int^{r},{ }_{H} H\right) \rightarrow \operatorname{Ext}_{H}^{d}\left(\int^{r},{ }_{H} k\right)
$$

is an isomorphism where $d$ is the injective dimension of $H$. We say $H$ satisfies (Cond2) if, for every simple left $H$-module $T \neq \int^{r}, \operatorname{Ext}_{H}^{d}(T, k)=0$. A $k$-algebra $A$ is called regular if gldim $A<\infty$ and called affine if it is finitely generated as a $k$-algebra. Algebras satisfying a polynomial identity are called PI algebras. Our first theorem is a generalization of Larson and Sweedler's result (GLD) $\Leftrightarrow$ (ITG), which is a homological version of Maschke's theorem in the infinite dimensional setting.

Theorem 0.1. Suppose $H$ is a noetherian affine PI Hopf algebra. Then $H$ is regular if and only if conditions (Cond1) and (Cond2) hold.

Theorem 0.1 follows from Theorems 3.3 and 3.4 (Cond1) is not as pretty as (ITG); but when $H$ is finite dimensional, namely, when $d=0$, (Cond1) is equivalent to (ITG) [Lemma 3.5. (Cond2) is even more strange; but when $H$ is finite dimensional, it is an easy consequence of (Cond1) [Lemma 3.5. Hence Theorem 0.1 generalizes the result of Larson and Sweedler (GLD) $\Leftrightarrow$ (ITG). When $H$ is commutative, it is also easy to see that (Cond2) is a consequence of (Cond1). Theorem 0.1 would become much nicer if one can show that (Cond2) is a consequence of (Cond1) in general.

The definition of homological integrals uses only the algebra structure of $H$, but not the coproduct of $H$. Any change of the coproduct of $H$ will not effect the homological integrals [Example 2.7. It is possible that there is another version of homological integrals that reflects the coalgebra structure of $H$.

In the finite dimensional case the left and right integrals live in the $H$. In the infinite dimensional case we will define the residue module $\Omega$ of $H$ where both the left and the right homological integrals live. We say $H$ is unimodular if $\int^{l}=\int^{r}$ in $\Omega$. We define the integral order of $H$, denoted by $i o(H)$, to be the minimal positive integer $n$ such that $\left(\int^{r}\right)^{\otimes n} \cong k$ as an $H$-bimodule [Definition 2.2. Then $H$ is unimodular if and only if $i o(H)=1$.

Integrals have been playing an important role in the studies of finite dimensional Hopf algebras. We expect that homological integrals will be useful in the research of infinite dimensional Hopf algebras of low Gelfand-Kirillov dimensions. The GelfandKirillov dimension is denoted by GK-dimension from now on. In the second half of the paper we use homological integrals to investigate the structure of regular Hopf algebras of GK-dimension one. Using the right homological integral of $H$ we can define a quotient Hopf algebra $H_{i q}$ of $H$, called the integral quotient of $H$ [Definition 4.2, which plays an important role in the study of regular Hopf algebras of GK-dimension one.

Theorem 0.2 (Theorem 7.1). Let $H$ be a noetherian affine Hopf algebra of GKdimension one and $H_{i q}$ be the integral quotient of $H$. Suppose $H$ is regular and prime. Then the following hold:

(a) $i o(H)\left(=\operatorname{dim}_{k} H_{i q}\right)=P I . \operatorname{deg}(H)$, where PI.deg is the PI degree.

(b) The coinvariant subalgebra $H^{\text {co } H_{i q}}$ is an affine commutative domain.

(c) If $H$ is unimodular (or equivalently $H_{i q}$ is trivial), then $H$ is commutative. If further $k$ is algebraically closed, then $H$ is isomorphic to either $k[x]$ or $k\left[x^{ \pm 1}\right]$. 
Partial results and conjectural descriptions are given when $H$ is not prime. Roughly speaking, every regular Hopf algebra $H$ of GK-dimension 1 should fit into a short exact sequence:

$$
0 \rightarrow H_{d i s} \rightarrow H \rightarrow H_{\text {conn }} \rightarrow 0
$$

where the connected component $H_{\text {conn }}$ is a regular prime Hopf quotient algebra of $H$ and the discrete component $H_{d i s}$ is conjecturally a finite dimensional subalgebra of $H$ [Theorem 6.5 and Remark 6.6]. The connected component $H_{\text {conn }}$ should fit into a short exact sequence:

$$
0 \rightarrow H_{c l} \rightarrow H_{\text {conn }} \rightarrow H_{i q} \rightarrow 0
$$

where the classical component $H_{c l}$ is the commutative subalgebra $H_{c o n n}^{c o H_{i q}}$ (see Theorem $0.2(\mathrm{~b}))$ and the integral quotient $H_{i q}$ is the dual of a finite group algebra acting on $H_{\text {conn }}$ [Theorem 7.1 and Remark 7.2. The statements can be found in Sections 6 and 7 .

Note that the conjectural descriptions of (E0.2.1) and (E0.2.2) are verified for group algebras. According to (E0.2.1) and (E0.2.2) every group $G$ of linear growth is isomorphic to $G_{d i s} \rtimes\left(\mathbb{Z} \rtimes G_{i q}\right)$ [Proposition 8.2. This description of groups of linear growth is well-known [IS, [St]. The ideas used here should be useful in further studies of homological properties and classification of infinite dimensional noetherian Hopf algebras and group algebras. For example we are wondering how the homological integrals can be used to study noetherian regular Hopf algebras of GK-dimension two. One of the testing questions is to extend Theorem 0.2 to the GK-dimension two case (see Examples 2.9 and 8.5).

Using a slight generalization of Theorem 0.1 (see Theorem 3.3(b)) and Theorem 0.2 we have the following easy corollary, which has a representation-theoretic flavor.

Corollary 0.3. Let $H$ be as in Theorem 0.2. If $M$ is a simple left $H$-module, then $\operatorname{dim}_{k} M$ divides io $(H)$.

Corollary 0.3 is proved at the end of Section 7 . Note that Corollary 0.3 fails for the GK-dimension two case [Examples 2.9 and 8.5.

Homological methods are effective for a large class of infinite dimensional Hopf algebras, in particular, for noetherian affine PI Hopf algebras. We refer to Bro, BG, WZ1, WZ2 for some known results and for questions concerning the homological properties of these Hopf algebras.

\section{Definition of INTEGRALS}

From now on let $k$ be a base field. There is no further restriction on $k$ unless otherwise stated. We refer to Montgomery's book [Mo for the basic definitions about Hopf algebras. Let $H$ be a Hopf algebra over $k$. Usually $k$ denotes the trivial algebra or the trivial Hopf algebra. For simplicity $k$ also denotes the trivial $H$-bimodule $H / \operatorname{ker} \epsilon$ where $\epsilon: H \rightarrow k$ is the counit of $H$. Usually we are working on left modules. Let $H^{\mathrm{op}}$ denote the opposite ring of $H$. A right $H$-module can be viewed as a left $H^{\mathrm{op}}$-module. An $H$-bimodule is sometimes identified with a left $H \otimes H^{\mathrm{op}}$-module where $\otimes$ denotes $\otimes_{k}$.

Recall that $H$ is Artin-Schelter Gorenstein (or AS-Gorenstein) if

(AS1) $\operatorname{injdim}_{H} H=d<\infty$,

(AS2) $\operatorname{dim}_{k} \operatorname{Ext}_{H}^{d}\left({ }_{H} k,{ }_{H} H\right)=1, \operatorname{Ext}_{H}^{i}\left({ }_{H} k,{ }_{H} H\right)=0$ for all $i \neq d$,

(AS3) the right $H$-module versions of the conditions (AS1,AS2) hold. 
We say $H$ is Artin-Schelter regular (or AS-regular) if it is AS-Gorenstein and it has finite global dimension.

It follows from the proof of [BG, Lemma 1.11] that (AS2) implies

$(\mathrm{AS} 2)^{\prime}$ for each finite dimensional simple left $H$-module $M, \operatorname{dim}_{\operatorname{Ext}_{H}^{d}}^{d}(M, H)=$ $\operatorname{dim} M$ and $\operatorname{Ext}_{H}^{i}(M, H)=0$ for all $i \neq d$; and the same holds for right modules.

Definition 1.1. Let $H$ be an AS-Gorenstein Hopf algebra of injective dimension d. Any nonzero element in $\operatorname{Ext}_{H}^{d}\left({ }_{H} k,{ }_{H} H\right)$ is called a left homological integral of $H$. We write $\int^{l}=\operatorname{Ext}_{H}^{d}\left({ }_{H} k,{ }_{H} H\right)$. Any nonzero element in $\operatorname{Ext}_{H \text { op }}^{d}\left(k_{H}, H_{H}\right)$ is called a right homological integral of $H$. We write $\int^{r}=\operatorname{Ext}_{H_{\text {op }}}^{d}\left(k_{H}, H_{H}\right)$. By abusing the language we also call $\int^{l}$ and $\int^{r}$ the left and the right homological integrals of $H$ respectively.

Homological integrals exist only for AS-Gorenstein Hopf algebras. Hence free Hopf algebras (of at least two variables) and universal enveloping algebras of infinite dimensional Lie algebras do not have homological integrals. On the other hand we expect that noetherian Hopf algebras are AS-Gorenstein, whence homological integrals exist. It is well-known that finite dimensional Hopf algebras are ASGorenstein of injective dimension 0. Affine noetherian PI Hopf algebras are ASGorenstein by [WZ1, Theorem 0.1]. Many Hopf algebras associated to classical and quantum groups are AS-Gorenstein and AS-regular [Bro, BG].

When $H$ is finite dimensional, then homological integrals agree with the classical integrals [Mo, Definition 2.1.1] in the following way: the (classical) left integral is an $H$-subbimodule of $H$; and it is identified with the left homological integral $\operatorname{Hom}_{H}(k, H)$ via the natural homomorphism

$$
\operatorname{Hom}_{H}(\epsilon, H): \operatorname{Hom}_{H}(k, H) \rightarrow \operatorname{Hom}_{H}(H, H) \cong H .
$$

The same holds for the right integral.

Note that both $\int^{l}$ and $\int^{r}$ are 1 -dimensional $H$-bimodules. As a left $H$-module, $\int^{l} \cong k$, but as a right $H$-module, $\int^{l}$ may not be isomorphic to $k$. A similar comment applies to $\int^{r}$.

Definition 1.2. Let $H$ be a Hopf algebra with $\int^{l}$ and $\int^{r}$. We say $H$ is unimodular if $\int^{l}$ is isomorphic to $k$ as $H$-bimodules.

The unimodular property means that

$$
h x=x h=\epsilon(h) x
$$

for all $h \in H$ and $x \in \int^{l}$. When $H$ is finite dimensional, this definition agrees with the classical definition in [Mo, p. 17].

Lemma 1.3. Suppose $H$ is noetherian. The following are equivalent:

(a) $H$ is unimodular.

(b) $\int^{r} \cong k$ as $H$-bimodules.

(c) $\int^{l} \cong \int^{r}$ as H-bimodules.

Proof. We only need to show that (a) $\Leftrightarrow$ (b). Since $H$ is noetherian and has finite injective dimension, there is a convergent spectral sequence [SZ, (3.8.1)]

$$
\operatorname{Ext}_{H}^{p}\left(\operatorname{Ext}_{H^{\text {op }}}^{q}\left(k_{H}, H_{H}\right),{ }_{H} H\right) \Longrightarrow k_{H} \text {. }
$$


The AS-Gorenstein condition gives rise to the isomorphism

$$
\operatorname{Ext}_{H}^{d}\left(\operatorname{Ext}_{H^{\text {op }}}^{d}\left(k_{H}, H_{H}\right),{ }_{H} H\right) \cong k_{H}
$$

where $d$ is the injective dimension of $H$. If (b) holds, then $\int^{r}:=\operatorname{Ext}_{H^{\text {op }}}^{d}\left(k_{H}, H_{H}\right) \cong$ $k$ as an $H$-bimodule. Hence (E1.3.1) implies that $\int^{l}:=\operatorname{Ext}_{H}^{d}\left({ }_{H} k,{ }_{H} H\right) \cong k$ as $H$-bimodule, whence $H$ is unimodular. By the left-right symmetry we have the other implication.

When $H$ is finite dimensional, $\int^{l}$ and $\int^{r}$ live in the same space $H$; and $H$ is unimodular if and only if $\int^{l}=\int^{r}$. This is also true for homological integrals after we define a residue module of $H$.

An $H$-module $M$ is locally finite if every finitely generated submodule of $M$ is finite dimensional over $k$. An $H$-bimodule is locally finite if it is locally finite on both sides. Let $\operatorname{Mod}_{f d} H$ (respectively, $\operatorname{Mod}_{f d} H^{\text {op }}$ ) denote the category of finite dimensional left (respectively, right) $H$-modules.

Definition 1.4. Let $H$ be AS-Gorenstein of injective dimension $d$. An $H$-bimodule $\Omega$ is called a residue module of $H$ if the following conditions hold:

(a) $\Omega$ is locally finite.

(b) The functors $\operatorname{Ext}_{H}^{d}\left(-,{ }_{H} H\right)$ and $\operatorname{Hom}_{H}\left(-,{ }_{H} \Omega\right)$ are naturally isomorphic when restricted to $\operatorname{Mod}_{f d} H \rightarrow \operatorname{Mod}_{f d} H^{\text {op }}$.

(c) The functors $\operatorname{Ext}_{H^{\text {op }}}^{d}\left(-, H_{H}\right)$ and $\operatorname{Hom}_{H^{\text {op }}}\left(-, \Omega_{H}\right)$ are naturally isomorphic when restricted to $\operatorname{Mod}_{f d} H^{\mathrm{op}} \rightarrow \operatorname{Mod}_{f d} H$.

Since $\Omega$ is locally finite, the above definition implies that $\Omega$ is unique up to a bimodule isomorphism. When $H$ is finite dimensional (namely, $d=0$ ), then $\Omega$ is isomorphic to $H$. The terminology "residue module" indicates that $\Omega$ is related to Yekutieli's residue complex [YZ].

Lemma 1.5. Suppose $\Omega$ is the residue module of $H$.

(a) The left and right homological integrals $\int^{l}$ and $\int^{r}$ can be identified with $\mathrm{H}$ subbimodules $\operatorname{Hom}_{H}\left({ }_{H} k,{ }_{H} \Omega\right)$ and $\operatorname{Hom}_{H \text { ор }}\left(k_{H}, \Omega_{H}\right)$ respectively. With this identification $\int^{l}$ and $\int^{r}$ live in a common vector space $\Omega$.

(b) $H$ is unimodular if and only if $\int^{l}=\int^{r}$.

Proof. (a) It follows from the natural $H$-bimodule homomorphisms

$$
\int^{l}:=\operatorname{Ext}_{H}^{d}(k, H) \cong \operatorname{Hom}_{H}(k, \Omega) \rightarrow \operatorname{Hom}_{H}(H, \Omega)=\Omega
$$

that $\int^{l}$ is a subbimodule of $\Omega$. The same argument works for $\int^{r}$.

(b) If $H$ is unimodular, then $\int^{l}=k x \cong k$ as $H$-bimodule, for some $x \in \Omega$. Since $\int^{r} \subset \Omega$ is the 1-dimensional $H$-subbimodule isomorphic to $k$ as right $H$-module, $\int^{r}=k x=\int^{l}$. The converse is trivial.

The existence of $\Omega$ is not all clear. We present some partial results here.

Let $H$ be an affine noetherian PI Hopf algebra. By [WZ1, Theorem 0.2(4)], there is an exact $H$-bimodule complex

$$
0 \rightarrow H \rightarrow I^{-d} \rightarrow \cdots \rightarrow I^{-1} \rightarrow I^{0} \rightarrow 0
$$

such that (E1.5.1) is a minimal injective resolution of the left $H$-module ${ }_{H} H$ and of the right $H$-module $H_{H}$ respectively. Further, when restricted to the left or the 
right, $I^{-i}$ is pure of GK-dimension $i$. The complex (E1.5.1) is called the residual complex of $H$ by Yekutieli [YZ. In the present paper we are mainly interested in the last term $I^{0}$. As a left (respectively, right) $H$-module, $I^{0}$ is a union of injective hulls of finite dimensional left (respectively, right) $H$-modules, which is locally finite. In general, a residual complex exists for every noetherian affine PI AS-Gorenstein algebra [YZ, Theorem 4.10].

Lemma 1.6. Suppose $H$ is a noetherian AS-Gorenstein Hopf algebra. If $H$ has a residual complex (E1.5.1), then $I^{0}$ is the residue module of $H$. In particular, the residue module $\Omega$ exists for every noetherian affine PI Hopf algebra.

Proof. Since the GK-dimension of $I^{0}$ is zero, it is locally finite. Another property of the residual complex is that $I^{-i}$ has no nonzero submodule of GK-dimension dimension less than $i$. If $i \neq 0$, then $\operatorname{Hom}_{H}\left(M, I^{-i}\right)=0$ for all finite dimensional left $H$-modules $M$. Thus

$$
\left.\operatorname{Hom}_{H}(M, \underline{E 1.5 .1})\right)=\operatorname{Hom}\left(M, I^{0}[-d]\right),
$$

which implies that

$$
\operatorname{Ext}_{H}^{d}\left(M,{ }_{H} H\right)=\operatorname{Hom}\left(M, I^{0}\right) .
$$

Similarly, $\operatorname{Ext}_{H^{\text {op }}}^{d}\left(N, H_{H}\right)=\operatorname{Hom}_{H^{\text {ор }}}\left(N, I_{H}^{0}\right)$ for all finite dimensional right $H$ modules $N$. By definition $I^{0}$ is the residue module.

Residual complexes may not exist for non-PI Hopf algebras [YZ, Remark 5.14]. But the residue module could still exist in that case. We mention one result without proof: the residue module exists for the enveloping algebra $U(\mathfrak{g})$ of a finite dimensional Lie algebra $\mathfrak{g}$.

Example 1.7. Let $\mathfrak{g}$ be the simple Lie algebra $s l_{2}$ generated by $e, f, h$ subject to relations

$$
[e, f]=h, \quad[h, e]=2 e, \quad[h, f]=-2 f .
$$

Let $H$ be the enveloping algebra $U(\mathfrak{g})$. Then $H$ is affine, noetherian and AS-regular with GK-dimension and global dimension 3.

It is well-known that $H$ has only one 1-dimensional simple module, which is the trivial module. Hence $\int^{l}=\int^{r}=k$ and $H$ is unimodular. It is not hard to verify that $\operatorname{Ext}_{H}^{i}(k, k)=0$ for all $i \neq 0,3$ and $\operatorname{Ext}_{H}^{i}(k, k) \cong k$ where $i=0,3$.

When char $k>0, H$ is PI and $\Omega$ is isomorphic to $\lim (H / I)^{*}$ where $I$ runs over all co-finite dimensional ideals of $H$. Here $(-)^{*}$ denotes the $k$-linear vector space dual. Incidently, $H^{\circ}$ is always defined to be $\lim (H / I)^{*}$. Here $H^{\circ}$ denotes the Hopf algebra dual in the sense of [Mo, Chapter 9].

When char $k=0, H$ is not PI and $\Omega$ is isomorphic to $\bigoplus_{n \geq 0} M_{n}(k)$, which is also equal to $\lim (H / I)^{*}$ where $I$ runs over all co-finite dimensional ideals of $H$.

In general one can prove that $U(\mathfrak{g})$ is unimodular if $\mathfrak{g}$ is a semisimple Lie algebra.

By Example 3.2, $U(\mathfrak{g})$ may not be unimodular, if $\mathfrak{g}$ is not semisimple.

\section{Order of Integral}

Since homological integrals agree with usual integrals in the finite dimensional case, we often use "integral" instead of "homological integral" from now on.

Let $\int^{l}$ and $\int^{r}$ be the left and the right integrals of $H$. For any $H$-bimodule $M$ let $S(M)$ denote the $H$-bimodule defined by the action

$$
h^{\prime} \cdot m \cdot h=S(h) m S\left(h^{\prime}\right)
$$


for all $m \in M, h, h^{\prime} \in H$. We can also define $S$ for one-sided $H$-modules in a similar way.

Lemma 2.1. Let $H$ be a Hopf algebra with integrals. Suppose the antipode $S$ of $H$ is bijective. Then $S\left(\int^{r}\right)=\int^{l}$ and $S\left(\int^{l}\right)=\int^{r}$.

Proof. Since $S$ is bijective, the functor $S$ is invertible. For any $H$-bimodules $M, N$, it is easy to check that

$$
\operatorname{Hom}_{H}(S(M), S(N)) \cong S\left(\operatorname{Hom}_{H_{\text {op }}}(M, N)\right)
$$

as $H$-bimodules. This isomorphism can be extended to their derived functors. Obviously, $S(k) \cong k$ and the map $S: H \rightarrow H$ induces an isomorphism $H \cong S(H)$ as right and left and $H$-bimodules. Hence we have

$$
\operatorname{Ext}_{H}^{d}(k, H) \cong \operatorname{Ext}_{H}^{d}(S(k), S(H)) \cong S\left(\operatorname{Ext}_{H^{\text {op }}}^{d}(k, H)\right) .
$$

Thus we proved $S\left(\int^{r}\right)=\int^{l}$. The proof of $S\left(\int^{l}\right)=\int^{r}$ is the same.

Given two left $H$-modules $M$ and $N$ we can define a left $H$-module structure on $M \otimes N$ via the coproduct $\Delta: H \rightarrow H \otimes H$. By the coassociativity of $\Delta$ the $n$th tensor product $M^{\otimes n}$ is a well-defined left $H$-module. Similarly we can define $\otimes$ for right $H$-modules. It is easy to check that $S(M \otimes N) \cong S(N) \otimes S(M)$ as right $H$-modules.

Definition 2.2. The integral order of $H$, denoted by $i o(H)$, is the order of the right integral $\int^{r}$, namely, the minimal positive integer $n$ (or $\infty$ if no such $n$ ) such that $\left(\int^{r}\right)^{\otimes n} \cong k$ as left $H$-modules.

The integral order has been used in the study of finite dimensional Hopf algebras either explicitly or implicitly. By the above definition $i o(H)$ seems dependent on the coproduct of $H$, because $\otimes$ is dependent on the coproduct of $H$. By Lemma 2.1 if the antipode $S$ is bijective, the $i o(H)$ can also be computed by using the left integral.

Any 1-dimensional left $H$-module $M$ can be identified as a quotient of an algebra homomorphism $\pi: H \rightarrow H /$ l.ann $H(M)$. Such an algebra homomorphism $\pi$ represents a group-like element in the dual Hopf algebra $H^{\circ}$. It is well-known that $\pi$ also defines an algebra automorphism $\sigma_{\pi}: H \rightarrow H$ given by

$$
\sigma_{\pi}: h \mapsto \sum h_{1} \pi\left(h_{2}\right),
$$

whose inverse is defined by

$$
\sigma_{\pi}^{-1}: h \mapsto \sum h_{1} \pi\left(S\left(h_{2}\right)\right) .
$$

Let $\sigma^{r}$ be the automorphism of $H$ induced by the map $\Sigma^{r}: H \rightarrow H / \operatorname{lann}\left(\int^{r}\right)$. The following is clear.

Lemma 2.3. Suppose integrals exist for $H$ :

(a) $i o(H)$ is equal to the order of the group-like element $\Sigma^{r} \in H^{\circ}$.

(b) io $(H)$ is equal to the order of the algebra automorphism $\sigma^{r}$ of $H$.

Next we want to investigate $i o(H)$ when $H$ is a noetherian PI ring.

Let $A$ be an algebra (not necessarily a Hopf algebra). We recall the definition of a clique in the prime spectrum $\operatorname{Spec} A$, and more details can be found in GW, Chapter 11]. Let $P$ and $Q$ be two prime ideals. If there is a nonzero $A$-bimodule $M$ 
that is a subquotient of $(P \cap Q) / P Q$ and is torsionfree as a left $A / P$-module and as a right $A / Q$-module. Then we say there is a link from $P$ to $Q$, written $P \leadsto Q$. The links make $\operatorname{Spec} A$ into a directed graph (i.e., a quiver) and the connected components of this graph are called cliques.

We will only work with noetherian affine PI algebras $A$. Let $M$ and $N$ be two simple left $A$-modules. We say $M$ and $N$ are in the same clique if $\operatorname{lann}_{A}(M)$ and $\operatorname{l.ann}_{A}(N)$ are in the same clique. It is easy to check that $l . a n n_{A}(M) \leadsto l . a n n_{A}(N)$ if and only if $\operatorname{Ext}_{A}^{1}(N, M) \neq 0$ (this is a consequence of [GW. Theorem 11.2]; see also [BW, p. 324]). In this case we sometimes write $M \leadsto N$. The following lemma is more or less known.

Lemma 2.4. Let $A$ be a noetherian affine PI algebra. Let $M$ and $N$ be two simple left $A$-modules. If $\operatorname{Ext}_{A}^{n}(M, N) \neq 0$ for some $n$, then $M$ and $N$ are in the same clique.

Proof. We prove a slightly more general statement, and the assertion follows from the general statement.

Claim: Let $X$ be any locally finite left $A$-module. Let

$$
0 \rightarrow X \rightarrow I^{0} \rightarrow I^{1} \rightarrow \cdots \rightarrow I^{n} \rightarrow \cdots
$$

be the minimal injective resolution of $X$. Then every simple subquotient of $I^{n}$ is in the same clique as some simple subquotient of $X$.

By induction we may assume that $n=0$. Let $S$ be a simple subquotient of $I^{0}$. Pick an element $x \in I^{0}$ such that the submodule $Y$ generated by $x$ has a quotient module isomorphic to $S$. If $Y \cap X$ is not in the kernel of $Y \rightarrow S$, then $S$ is a quotient of $Y \cap X$. We are done. Otherwise, $Y / Y \cap X \rightarrow S$ is surjective. In this case by the induction on $\operatorname{dim}_{k} Y / Y \cap X$ we can assume that $Y / Y \cap X$ is isomorphic to $S$ (note that we can also change $X$ when using this induction). Since $Y$ is an essential extension of $Y \cap X$, we have $\operatorname{Ext}_{A}^{1}(S, Y \cap X) \neq 0$. This implies that there is a simple subquotient $S^{\prime}$ of $Y \cap X$ such that $\operatorname{Ext}_{A}^{1}\left(S, S^{\prime}\right) \neq 0$. Hence $S$ and $S^{\prime}$ are linked and we have proved our claim.

Now let $X$ be the simple module $N$. If $\operatorname{Ext}_{A}^{n}(M, N) \neq 0$, then $M$ is a subquotient of $I^{n}$ where $I^{n}$ is the $n$th term in the minimal injective resolution of $N$. By the claim $M$ and $N$ are in the same clique.

Proposition 2.5. Let $H$ be an affine noetherian PI Hopf algebra. Suppose that $\operatorname{Ext}_{H}^{i}\left(\int^{r}, k\right) \neq 0$ for some $i$. If the clique containing $k$ is finite, then $i o(H)$ is finite.

Proof. Since $\int^{r}$ is 1-dimensional, by [WZ2, Lemma 1.3]

$$
\left(S\left(\int^{r}\right)\right)^{*} \otimes \int^{r} \cong k \cong \int^{r} \otimes\left(S\left(\int^{r}\right)\right)^{*}
$$

as left $H$-modules. Note that our definition of * is slightly different from the definition given in WZ2, p. 602] and that's the reason we need to add $S$. The equation (E2.5.1) implies that the functor $\int^{r} \otimes-$ is an auto-equivalence of the category of left $H$-modules. Hence $\operatorname{Ext}_{H}^{i}(M, N) \cong \operatorname{Ext}_{H}^{i}\left(\int^{r} \otimes M, \int^{r} \otimes N\right)$ for all left $H$-modules $M, N$ and for all $i$. In particular,

$$
\operatorname{Ext}_{H}^{i}\left(\left(\int^{r}\right)^{\otimes(p+1)},\left(\int^{r}\right)^{\otimes p}\right) \cong \operatorname{Ext}_{H}^{i}\left(\int^{r}, k\right) \neq 0
$$

for all $p$. By Lemma 2.4 all $\left(\int^{r}\right)^{\otimes p}$ 's are in the clique containing $k$. Since that clique is finite by hypotheses, there are $n>m$ such that $\left(\int^{r}\right)^{\otimes n} \cong\left(\int^{r}\right)^{\otimes m}$. Hence $\left(\int^{r}\right)^{\otimes(n-m)} \cong k$ and $i o(H) \leq n-m$. 
Lemma 2.6. Let $H$ be an AS-Gorenstein Hopf algebra and let $x$ be a normal nonzero-divisor of $H$ such that $(x)$ is a Hopf ideal of $H$. Suppose that $\tau$ is the algebra automorphism of $H$ such that $x h=\tau(h) x$ for all $h \in H$.

(a) $H^{\prime}:=H /(x)$ is an AS-Gorenstein Hopf algebra.

(b) $\int_{H}^{l} \cong\left(\int_{H^{\prime}}^{l}\right)^{\tau^{-1}}$ as right $H$-modules.

(c) If $x$ is central, then $\int_{H}^{l} \cong \int_{H^{\prime}}^{l}$ and $i o(H)=i o\left(H^{\prime}\right)$.

Proof. Let $M$ be a left $H^{\prime}$-module. By change of rings [Ro, Theorem 11.66 or Corollary 11.68], we have

$$
\operatorname{Ext}_{H}^{p}(M, H) \cong \operatorname{Ext}_{H}^{p}\left(H /(x) \otimes_{H /(x)} M, H\right) \cong \operatorname{Ext}_{H /(x)}^{p-1}\left(M, \operatorname{Ext}_{H}^{1}(H /(x), H)\right) .
$$

An easy computation shows that $\operatorname{Ext}_{H}^{1}(H /(x), H) \cong \tau(H /(x)) \cong(H /(x))^{\tau^{-1}}$. Hence

$$
\operatorname{Ext}_{H}^{p}(M, H) \cong \operatorname{Ext}_{H /(x)}^{p-1}\left(M,(H /(x))^{\tau^{-1}}\right) \cong \operatorname{Ext}_{H /(x)}^{p-1}(M, H /(x))^{\tau^{-1}} .
$$

This shows that $\operatorname{injdim} H^{\prime} \leq \operatorname{injdim} H-1$. Let $M=k$. Then we see that $\operatorname{injdim} H^{\prime}=\operatorname{injdim} H-1$ and $\int_{H}^{l}=\left(\int_{H^{\prime}}^{l}\right)^{\tau^{-1}}$ as right $H$-modules. The ASGorenstein property follows from this isomorphism. Finally when $x$ is central, then $\tau$ is the identity map of $H$ and $\int_{H}^{l}=\int_{H^{\prime}}^{l}$. In this case $i o(H)=i o\left(H^{\prime}\right)$.

We will have a finiteness result about $i o(H)$ in Lemma 5.3(g). To conclude this section we give two examples, the first of which comes from Taft's construction [Ta] (see also [Mo, Example 1.5.6]).

Example 2.7. Let $n, m$ and $t$ be integers and $\xi$ be an $n$th primitive root of 1 . Let $H$ be the $k$-algebra generated by $x$ and $g$ subject to the relations

$$
g^{n}=1, \quad \text { and } \quad x g=\xi^{m} g x .
$$

So $H$ is commutative if and only if $\xi^{m}=1$. The coalgebra structure of $H$ is defined by

$$
\Delta(g)=g \otimes g, \epsilon(g)=1 \quad \text { and } \quad \Delta(x)=x \otimes 1+g^{t} \otimes x, \epsilon(x)=0 .
$$

So $H$ is cocommutative if and only if $g^{t}=1$. The antipode $S$ of $H$ is defined by

$$
S(g)=g^{-1} \text { and } S(x)=-g^{-t} x=-\xi^{m t} x g^{-t} .
$$

The order of the antipode $S$ is $2 \operatorname{order}\left(\xi^{m t}\right)$. Hence $H$ is involutive if and only if $\xi^{m t}=1$. One way to understand the algebra structure of $H$ is to view it as a smash product $k[x] \# k G$ where $G$ is the group of $\langle g\rangle$. The $k G$ action on $k[x]$ is determined by

$$
g \circ x=\xi^{m} x .
$$

When char $k$ does not divide $n$, by [LL, Corollary 2.4], the global dimension of $H$ is bounded by the global dimension of $k[x]$. Hence gldim $H=1$. Since $x^{n}$ is a central element, $H$ is finite over its center. Therefore $H$ is AS-regular. Changing $t$ to another $t^{\prime}$ will only change the coproduct of $H$, but not the algebra structure of $H$. So this change will not effect the (homological) integrals of $H$. To compute the integrals we use Lemma 2.6. Note that $x$ is a normal element with $x h=\tau(h) x$ for all $h \in H$ and where $\tau: x \rightarrow x, g \rightarrow \xi^{m} g$. Since $H^{\prime}=H /(x)$ is isomorphic to $k G$, it is commutative and finite dimensional. Hence $\int_{H^{\prime}}^{l}=k=H /(x, g-1)$. By Lemma 2.6. $\int_{H}^{l}=H /(x, g-1)^{\tau^{-1}} \cong H /\left(x, g-\xi^{-m}\right)$ as right $H$-modules. By Lemma 2.1. $\int^{r} \cong H /\left(x, g-\xi^{m}\right)$ as left $H$-modules. 
The integral order $i o(H)$ is equal to the $\operatorname{order}\left(\xi^{m}\right)$. When $\operatorname{gcd}(m, n)=1$, then $H$ is a prime ring of PI degree $n$. In this case the PI degree of $H$ is equal to $i o(H)$. In this example, any change of coproduct of $H$ does not effect $i o(H)$ though the definition of $i o(H)$ uses the coproduct of $H$.

If $\xi^{m t}=-1$, then the ideal generated by $x^{2}$ is a Hopf ideal. The quotient Hopf algebra is not semisimple because the order of $S$ is 4 . So a quotient Hopf algebra of a regular algebra may not be regular. On the other hand, certain quotient Hopf algebras of a regular algebras are regular if they have the same GK-dimension; see Lemma 5.5 (a).

Lemma 2.8. Let $H$ and $K$ be two noetherian AS-Gorenstein Hopf algebra such that $H \otimes K$ is noetherian with finite injective dimension. Then

(a) $H \otimes K$ is AS-Gorenstein and injdim $H \otimes K=\operatorname{injdim} H+\operatorname{inj} \operatorname{dim} K$.

(b) $\int_{H \otimes K}^{r}=\int_{H}^{r} \otimes \int_{K}^{r}$ and $i o(H \otimes K)=\operatorname{lcm}\{i o(H), i o(K)\}$.

Proof. Since $k_{H \otimes K}=k_{H} \otimes k_{K}$, we have

$$
\operatorname{Ext}_{H \otimes K}^{i}(k, H \otimes K) \cong \bigoplus_{j=0}^{i} \operatorname{Ext}_{H}^{j}(k, H) \otimes \operatorname{Ext}_{K}^{i-j}(k, K) .
$$

The assertions follow from the AS-Gorenstein property of $H$ and $K$.

Example 2.9. Let $H$ be the Hopf algebra in Example 2.7 with $m=t=1$ and $n>$ 1. Then $H$ is prime and $i o(H)=P I \cdot \operatorname{deg}(H)=n$. It is easy to check that $H \otimes H$ is prime, noetherian, affine PI of injective dimension 2 and that PI.deg $(H \otimes H)=n^{2}$. By Lemma 2.8 $i o(H \otimes H)=n$. Since PI.deg $(H \otimes H)=n^{2}$, there is a simple $H \otimes H$-module of dimension $n^{2}$. Therefore Corollary 0.3 fails for Hopf algebras of GK-dimension 2.

In general, $i o(K) \neq P I$.deg $(K)$ for a nice Hopf algebra $K$ of GK-dimension $>1$. Theorem 0.2 fails badly for higher GK-dimensions.

\section{Extension of LARSON-SweEdLER}

As mentioned in the introduction, Larson and Sweedler proved the following version of Maschke's theorem: a finite dimensional Hopf algebra is semisimple artinian (i.e., has global dimension 0) if and only if $\epsilon\left(\int^{l}\right) \neq 0$, and if and only if $\epsilon\left(\int^{r}\right) \neq 0$. The terms $\epsilon\left(\int^{l}\right)$ and $\epsilon\left(\int^{r}\right)$ make sense in the infinite dimensional case in the following way. The counit $\epsilon: H \rightarrow k$ induces an $H$-bimodule homomorphism, which is also denoted by $\epsilon$,

$$
\epsilon: \int^{l}=\operatorname{Ext}_{H}^{d}\left({ }_{H} k,{ }_{H} H\right) \rightarrow \operatorname{Ext}_{H}^{d}\left({ }_{H} k,{ }_{H} k\right) .
$$

Lemma 3.1. If $\epsilon\left(\int^{l}\right) \neq 0$, then $H$ is unimodular.

Proof. Note that $\operatorname{Ext}_{H}^{d}\left({ }_{H} k,{ }_{H} k\right)$ is isomorphic to a direct sum of $k$ as $H$-bimodules. If $\epsilon\left(\int^{l}\right) \neq 0$, then $\epsilon$ in (E3.0.1) embeds $\int^{l}$ into $\operatorname{Ext}_{H}^{d}\left({ }_{H} k,{ }_{H} k\right)$. Hence $\int^{l}$ is isomorphic to $k$.

To generalize Larson-Sweedler's result it is natural to ask if the condition $\epsilon\left(\int^{l}\right) \neq$ 0 in Lemma 3.1 is equivalent to $H$ having finite global dimension. The answer is "No", as the next example shows (see also Example 2.7). 
Example 3.2. Let $L$ be the 2-dimensional solvable Lie algebra generated by $x$ and $y$ subject to the relation $[x, y]=x$. Let $H$ be the enveloping algebra $U(L)$. Then $H$ is a noetherian affine domain of global dimension 2. It is involutory, i.e., $S^{2}=1$. If char $k \neq 0$, then it is a PI algebra.

The trivial module $k$ is isomorphic to $H /(x, y)$. By a computation in ASZ, Example 3.2] or using Lemma 2.6 we have

$$
\int^{l}:=\operatorname{Ext}_{H}^{2}\left({ }_{H} k,{ }_{H} H\right) \cong H /(x, y+1) \not k
$$

as right $H$-modules. For every $p,\left(\int^{l}\right)^{\otimes p} \cong H /(x, y+p)$ as right $H$-modules. Hence $i o(H)=\operatorname{char} k$ if char $k \neq 0$ or $i o(H)=\infty$ otherwise. In particular, $H$ is not unimodular. By Lemma 3.1, $\epsilon\left(\int^{l}\right)=0$.

Conditions (Cond1) and (Cond2) are the correct replacement of (ITG), as the next result shows. Recall from the introduction that $H$ satisfies (Cond1) if the map

$$
\operatorname{Ext}_{H}^{d}\left(\int^{r}, \epsilon\right): \operatorname{Ext}_{H}^{d}\left(\int^{r},{ }_{H} H\right) \rightarrow \operatorname{Ext}_{H}^{d}\left(\int^{r}, k\right)
$$

is an isomorphism where $d$ is the injective dimension of $H$ and that $H$ satisfies (Cond2) if for every simple left $H$-module $T \not \int^{r}, \operatorname{Ext}_{H}^{d}(T, k)=0$.

Theorem 3.3. Suppose $H$ is noetherian and AS-regular of global dimension d.

(a) (Cond1) and (Cond2) hold.

(b) Let $W$ be a finite dimensional simple left $H$-module and let $W^{\prime}$ denote the simple left $H$-module $\operatorname{Ext}_{H}^{d}(W, H)^{*}$. Then, for every surjective map $H \rightarrow$ $W^{\prime}$ of left $H$-modules, the induced map $\operatorname{Ext}_{H}^{d}(W, H) \rightarrow \operatorname{Ext}_{H}^{d}\left(W, W^{\prime}\right)$ is nonzero and surjective. Further, for every simple left $H$-module $T \nRightarrow W$, $\operatorname{Ext}_{H}^{d}\left(T, W^{\prime}\right)=0$.

Proof. If $W=\int^{r}$, then $W^{\prime}=\operatorname{Ext}_{H}^{d}\left(\int^{r}, H\right)^{*} \cong\left(k_{H}\right)^{*} \cong{ }_{H} k$ (see Lemma 1.3 for the argument of $\left.\operatorname{Ext}_{H}^{d}\left(\int^{r}, H\right) \cong k_{H}\right)$. Also $\operatorname{Ext}_{H}^{d}\left(\int^{r}, H\right)$ is 1-dimensional, and the assertion (b) implies (a). So we only prove (b).

By [ASZ, Proposition 7.1], for every finite dimensional $H$-module $W$ and every noetherian left $H$-module $N$ there is a natural isomorphism

$$
\operatorname{Ext}_{H}^{d-i}(W, N) \cong \operatorname{Ext}_{H}^{i}\left(N, W^{\prime}\right)^{*}
$$

where $W^{\prime}=\operatorname{Ext}_{H}^{d}(W, H)^{*}$ and where $*$ is the $k$-linear dual. For $i=0$, the functor $\operatorname{Ext}_{H}^{d}(W,-)$ is equivalent to $\operatorname{Hom}_{H}\left(-, W^{\prime}\right)^{*}$. This equivalence translates the assertions in (b) into the following assertions about $\operatorname{Hom}_{H}\left(-, W^{\prime}\right)$ :

(i) the induced map $\operatorname{Hom}_{H}\left(H, W^{\prime}\right)^{*} \rightarrow \operatorname{Hom}_{H}\left(W^{\prime}, W^{\prime}\right)^{*}(\cong k)$ is surjective; and

(ii) $\operatorname{Hom}_{H}(W, T)=0$ for all simple $T \neq W$.

Both (i) and (ii) are obviously true now.

Theorem 3.3 gives one implication in Theorem 0.1 . In the rest of this section we prove the other implication in Theorem 0.1 . We introduce a condition slightly weaker than those in Theorem 3.3. We say $H$ satisfies (Cond3) if, for every simple left $H$-module $T$,

$$
\operatorname{Ext}_{H}^{d}(T, \epsilon): \operatorname{Ext}_{H}^{d}\left(T,{ }_{H} H\right) \rightarrow \operatorname{Ext}_{H}^{d}\left(T,{ }_{H} k\right)
$$

is surjective. It is obvious that (Cond 3$)$ is a consequence of $(\mathrm{Cond} 1)+(\mathrm{Cond} 2)$ (when taking $W=\int^{r}$ and $W^{\prime \prime}=k$ ). The following is a converse of Theorem 3.3. 
Theorem 3.4. Let $H$ be noetherian and AS-Gorenstein. Suppose that injective hulls of finite dimensional left $\mathrm{H}$-modules are locally finite. If (Cond3) holds, then $H$ is regular.

Combining Theorems 3.3 and 3.4 , we see that $H$ is regular if and only if (Cond3) holds.

Proof of Theorem 3.4. Let $d$ be the injective dimension of $H$. First we prove the following two statements:

(a) $\operatorname{Ext}_{H}^{d}\left(-,{ }_{H} k\right)$ is a right exact functor on $\operatorname{Mod}_{f d} H$.

(b) $\operatorname{Ext}_{H}^{d}\left(M,{ }_{H} H\right) \rightarrow \operatorname{Ext}_{H}^{d}\left(M,{ }_{H} k\right)$ is surjective for all $M \in \operatorname{Mod}_{f d} H$.

Consider an exact sequence in $\operatorname{Mod}_{f d} H$,

$$
0 \rightarrow L \rightarrow M \rightarrow N \rightarrow 0
$$

with nonzero $L$ and $N$. Applying $\operatorname{Ext}_{H}^{d}(-, H)$ and $\operatorname{Ext}_{H}^{d}(-, k)$ to (E3.4.1) we obtain a row exact commutative diagram

(E3.4.2)

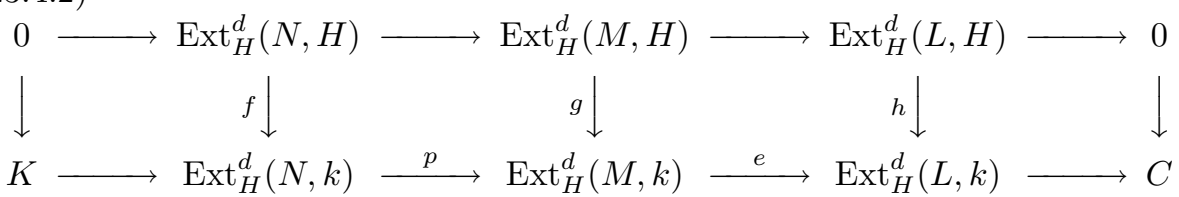

for some modules $K$ and $C$. The top row is exact since $H$ is AS-Gorenstein. The bottom row is exact for $K=\operatorname{ker}(p)$ and $C=\operatorname{coker}(e)$.

We first prove (b) by induction on $\operatorname{dim}_{k} M$. By (Cond3) the assertion (b) holds when $M$ is simple (in particular when $\operatorname{dim}_{k} M=1$ ). If $\operatorname{dim}_{k} M>1$ and $M$ is not simple, then we can find nonzero $L$ and $N$ that fit into (E3.4.1). By induction hypothesis the vertical maps $f$ and $h$ are surjective. By a diagram chasing or by [Ro, Lemma 3.32 (five lemma)], $g$ is surjective. Then (b) follows from induction.

Now assume (b). To prove (a) we need to show that the map $e$ is surjective. This is clear since $g$ and $h$ are surjective by (b).

Secondly we show that (a) implies that ${ }_{H} k$ has finite injective dimension. Let

$$
0 \rightarrow{ }_{H} k \rightarrow J^{0} \rightarrow J^{1} \rightarrow \cdots \rightarrow J^{d} \rightarrow J^{d+1} \rightarrow \cdots
$$

be a minimal injective resolution of ${ }_{H} k$.

Suppose that $k$ has infinite injective dimension. Then $J^{d+1} \neq 0$. Let $T=$ $\operatorname{ker}\left(J^{d} \rightarrow J^{d+1}\right)$. Since (E3.4.3) is minimal, $T$ is an essential proper submodule of $J^{d}$. Let $\Phi$ be the set of all co-finite dimensional ideals of $H$. For every $I \in \Phi$, let

$$
T_{I}=\operatorname{Hom}_{H}(H / I, T) \text { and } J_{I}=\operatorname{Hom}_{H}\left(H / I, J^{d}\right) .
$$

Since $H$ is noetherian, $\operatorname{Ext}_{H}^{d}(M, k)$ is finite dimensional over $k$ for every finitely generated left $H$-module $M$. When $M$ is finite dimensional and simple, $J^{d}$ contains only finitely many copies of $M$ for each such $M$. For a fixed $I \in \Phi$, the socle of $J_{I}$ is finite dimensional since there are finitely many simple modules over $H / I$. This implies that $J_{I}$ is finite dimensional. Since $J^{d}$ is locally finite, we have

$$
T=\lim _{I \in \Phi} T_{I} \quad \text { and } \quad J^{d}=\lim _{I \in \Phi} J_{I} .
$$

Then the fact $T \neq J^{d}$ implies that $T_{I} \neq J_{I}$ for some $I$. Now we consider the exact sequence of finite dimensional left $H$-modules

$$
0 \rightarrow T_{I} \rightarrow J_{I} \rightarrow N \rightarrow 0
$$


where $N=J_{I} / T_{I}$ is nonzero. Because any map $f: T_{I} \rightarrow T$ factoring through $J^{d-1} \rightarrow T$ cannot be injective, the inclusion map $\alpha: T_{I} \rightarrow T$ induces a nonzero element

$$
\bar{\alpha} \in \operatorname{Ext}_{H}^{d}\left(T_{I}, k\right)=\operatorname{Hom}_{H}\left(T_{I}, T\right) / \operatorname{im}\left(\operatorname{Hom}_{H}\left(T_{I}, J^{d-1}\right)\right) .
$$

We claim that there is no element

$$
\bar{\beta} \in \operatorname{Ext}_{H}^{d}\left(J_{I}, k\right)=\operatorname{Hom}_{H}\left(J_{I}, T\right) / \operatorname{im}\left(\operatorname{Hom}_{H}\left(J_{I}, J^{d-1}\right)\right)
$$

such that

$$
\bar{\alpha}=\bar{\beta} \circ i
$$

where $i: T_{I} \rightarrow J_{I}$ is the inclusion. The equation (E3.4.4) means that there is a map $\beta \in \operatorname{Hom}_{H}\left(J_{I}, T\right)$ such that $\alpha=\beta \circ i+\partial^{d-1} \circ \phi$ where $\phi$ is some element in $\operatorname{Hom}_{H}\left(T_{I}, J^{d-1}\right)$ and $\partial^{d-1}: J^{d-1} \rightarrow J^{d}$ is the differential map of the complex (E3.4.3). To show the claim we rewrite the equation as $\alpha-\partial^{d-1} \circ \phi=\beta \circ i$. For any simple submodule $S$ of $T_{I}$, the image $\phi(S)$ must be in the socle of $J^{d-1}$. Hence $\partial^{d-1} \circ \phi(S)=0$ since $(\mathrm{E} 3.4 .3)$ is minimal. This implies that the map $\alpha^{\prime}:=\alpha-\partial^{d-1} \circ \phi$ is injective on the socle of $T_{I}$, whence injective on $T_{I}$. Since the socle of $T_{I}$ is equal to the socle of $J_{I}$ by minimality of (E3.4.3), the equation $\alpha^{\prime}=\beta \circ i$ implies that the map $\beta$ is injective on the socle of $J_{I}$, whence injective on $J_{I}$. But this is impossible because $\operatorname{dim} J_{I}>\operatorname{dim} T_{I}$. Therefore $\beta$ does not exist.

Nonexistence of $\beta$ shows that the sequence

$$
\rightarrow \operatorname{Ext}_{H}^{d}(N, k) \rightarrow \operatorname{Ext}_{H}^{d}\left(J_{I}, k\right) \rightarrow \operatorname{Ext}_{H}^{d}\left(T_{I}, k\right) \rightarrow 0
$$

is not right exact. This yields a contradiction with (a). This contradiction shows that ${ }_{H} k$ has finite injective dimension.

Since $H$ is noetherian and has finite injective dimension, $H$ itself serves as a dualizing complex over $H$. By WZ3, Lemma 2.1], the right $H$-module $\int^{l}:=$ $\operatorname{Ext}_{H}^{d}(k, H) \cong \operatorname{RHom}_{H}(k, H)[d]$ (where $[d]$ is the $d$ th complex shift) has finite right projective dimension. Since $\int^{l}$ is 1-dimensional, by [WZ2, Proposition 1.4], gldim $H$ is finite.

Theorem 0.1 follows from Theorems 3.3 and 3.4 because injective hulls of finite dimensional modules over noetherian affine PI rings are locally finite.

Suppose $H$ is commutative. Then for any two nonisomorphic simple modules $S_{i}$, we have $\operatorname{Ext}_{H}^{i}\left(S_{1}, S_{2}\right)=0$. This implies that $\operatorname{Ext}_{H}^{i}(S, k)=0$ for all $i$ all $S \neq k$. Since $H$ is commutative, $\int^{r}=\int^{l}=k$. Hence (Cond2) is automatic. A similar statement holds for finite dimensional Hopf algebras.

Lemma 3.5. Suppose $H$ is finite dimensional. Then the following are equivalent:

(a) (ITG).

(b) (Cond1).

(c) (Cond1) plus (Cond2).

Proof. It's easy to see that (ITG) implies that $H$ is unimodular. The same applies to (Cond1). So we might as well assume $H$ is unimodular. Under this hypothesis, (Cond2) becomes trivial, and (ITG) is just (Cond1).

Based on the limited evidences in the finite dimensional case and in the commutative case we ask the following question. 
Question 3.6. Let $H$ be a noetherian affine PI Hopf algebra of injective dimension $d$. If (Cond1) holds, is $H$ then regular?

\section{Integral QUOTIEnT OF $H$}

In this section we discuss some quotient (i.e., factor) Hopf algebras of $H$ which will be used later. The following is clear.

Lemma 4.1. Let $H$ be a Hopf algebra and let $\mathfrak{m}$ be an ideal of $H$. Then there is a unique maximal Hopf ideal $J$ contained in $\mathfrak{m}$.

Definition 4.2. Let $\int^{r}$ be the right integral of $H$. Let $\mathfrak{m}=l . a n n_{H}\left(\int^{r}\right)$ and let $J_{i q}$ be the maximal Hopf ideal contained in $\mathfrak{m}$. The quotient Hopf algebra $H / J_{i q}$ is called the integral quotient of $H$, denoted by $H_{i q}$.

Another Hopf quotient related to $H_{i q}$ is the abelianization of $H$. The following lemma is clear.

Lemma 4.3. Let $H$ be a Hopf algebra. Let I be the ideal generated by $x y-y x$ for all $x, y \in H$. Then $I$ is a Hopf ideal and $H \rightarrow H / I$ is a Hopf algebra homomorphism.

We call $H / I$ in the above lemma the abelianization of $H$, denoted by $H_{a b}$. Let $\pi$ be the canonical Hopf algebra homomorphism $H \rightarrow H_{a b}$. If $f: H \rightarrow H^{\prime}$ is a Hopf algebra homomorphism with $H^{\prime}$ being commutative, then $f$ factors through $\pi: H \rightarrow H_{a b}$. As in Example 7.3 the map $\pi: H \rightarrow H_{a b}$ is not necessarily conormal in the sense of [Mo, Definition 3.4.5]. Since $\mathfrak{m}:=\operatorname{lann}_{H}\left(\int^{r}\right)$ has co-dimension 1, $I \subset \mathfrak{m}$. This implies that $I \subset J$. Therefore $H_{i q}$ is a Hopf quotient of $H_{a b}$. As a consequence $H_{i q}$ is a commutative Hopf algebra.

Lemma 4.4. Let $H$ be a Hopf algebra with integrals.

(a) io $(H)$ is finite if and only if $\operatorname{dim}_{k} H_{i q}$ is finite.

(b) If io $(H)=n<\infty$, then $H_{i q} \cong\left(k \mathbb{Z}_{n}\right)^{\circ}$.

(c) $J_{i q}=\bigcap_{i=0}^{n-1} \operatorname{ker}\left(\left(\Sigma^{r}\right)^{i}: H \rightarrow k\right)$.

Proof. Suppose first $H_{i q}$ is finite dimensional. Let $\Sigma^{r}: H \rightarrow H / \mathfrak{m}$ where $\mathfrak{m}=$ $\operatorname{l.ann}_{H}\left(\int^{r}\right)$. Then $\Sigma^{r}$ is a group-like element in $H_{i q}^{\circ} \subset H^{\circ}$. Hence $\bigoplus_{i \in \mathbb{Z}} k\left(\Sigma^{r}\right)^{i}$ is a Hopf subalgebra of $H_{i q}^{\circ}$. By Lemma2.3(a) $i o(H)$ is bounded by $\operatorname{dim}_{k} H_{i q}^{\circ}=\operatorname{dim} H_{i q}$.

Now assume that $i o(H)=n<\infty$. Then the finite group algebra $\bigoplus_{i=0}^{n-1} k\left(\Sigma^{r}\right)^{i}$ is a Hopf subalgebra of $H_{i q}^{\circ}$. Hence $I:=\bigcap_{i=0}^{n-1} \operatorname{ker}\left(\left(\Sigma^{r}\right)^{i}: H \rightarrow k\right)$ is a Hopf ideal of $H$. Since $\operatorname{ker}\left(\Sigma^{r}: H \rightarrow k\right)=$ l.ann $n_{H}\left(\int^{r}\right)=\mathfrak{m}$ and since $\mathfrak{m}$ contains the Hopf ideal $J_{i q}$, by a computation using coproduct, each $\operatorname{ker}\left(\left(\Sigma^{r}\right)^{i}: H \rightarrow k\right)$ contains $J_{i q}$. Hence we have $I \supset J_{i q}$. By the maximality of $J_{i q}$, we have $I=J_{i q}$. Thus $\operatorname{dim}_{k} H_{i q}=n$. This forces that $H_{i q}^{\circ}=\bigoplus_{i=0}^{n-1} k\left(\Sigma^{r}\right)^{i}$. The assertions follow.

Let $G$ be a group. Define $G_{a b}$ to be $G /[G, G]$. The following lemma holds because $H_{i q}$ is a Hopf quotient of $H_{a b}$.

Lemma 4.5. Suppose integrals exist for $H$. If $H_{a b}$ is finite dimensional, then $i o(H)$ divides $\operatorname{dim} H_{a b}$. If $H$ is a group algebra $k G$, then io $(H)$ divides $\left|G_{a b}\right|$.

The next example shows that $H_{i q} \neq H_{a b}$ in general even when both are finite dimensional. 
Example 4.6. Let $\mathbb{D}$ denote the group $\left\langle g, x \mid g^{2}=1, g x g=x^{-1}\right\rangle$. It is easy to see that $\mathbb{D}$ contains $\mathbb{Z}$ (generated by $x$ ) as a normal subgroup and $\mathbb{D} / \mathbb{Z} \cong \mathbb{Z}_{2}$. Also $\mathbb{D}$ is isomorphic to the free product $\mathbb{Z}_{2} * \mathbb{Z}_{2}$. The Hopf algebra $k \mathbb{D}$ (when char $k \neq 2$ ) is prime and regular of GK-dimension 1 . The abelianization $H_{a b}$ is $H /\left(x^{2}-1\right)$, and isomorphic to $k\left(\mathbb{Z}_{2} \oplus \mathbb{Z}_{2}\right)$.

To compute the integral, we note that $x-1$ is a normal element of $H$ since

$$
(x-1) g=\left(-g x^{-1}\right)(x-1) .
$$

Using this fact and Lemma2.6 one sees that $\int^{r} \cong H /(x-1, g+1)$ as left $H$-modules. Hence $i o(H)=2$ and $H_{i q}=H /(x-1) \cong k \mathbb{Z}_{2}$. Therefore $H_{a b} \neq H_{i q}$.

We will also use the following easy lemma.

Lemma 4.7. Let $I$ be any Hopf ideal of H. Let $J=\bigcap_{n} I^{n}$. Then $J$ is a Hopf ideal of $H$.

\section{Noetherian PI Hopf ALgebras}

The first step beyond the finite dimensional case is to look into affine noetherian Hopf algebras of GK-dimension 1. By [SSW], such an algebra is PI. In this section we collect some known results about noetherian affine PI Hopf algebras. In later sections we will concentrate on Hopf algebras of GK-dimension one.

Lemma 5.1. Let $H$ be a noetherian affine PI Hopf algebra of GK-dimension d.

(a) $H$ is AS-Gorenstein of injective dimension d. As a consequence, the left and right integrals of $H$ exist.

(b) $H$ has a quasi-Frobenius ring of fractions, denoted by $Q$.

(c) The residue module of $H$ exists.

(d) If $d=1$, the injective module $Q / H$ is the residue module of $H$.

Proof. (a) This is [WZ1, Theorem 0.1].

(b) This is WZ1, Theorem 0.2(2)].

(c,d) This follows from [WZ1, Theorem 0.2(4)] and Lemma 1.6.

The following lemma is a consequence of [WZ2, Theorem 0.1 and Corollary 3.7].

Lemma 5.2. Let $H$ be a noetherian affine PI Hopf algebra. Suppose that the base field $k$ is of characteristic zero and that $H$ is involutive. If either $H$ is finite over its affine center or GKdim $H=1$, then $H$ is regular.

The following lemma is a consequence of [SZ, Theorems 5.4, 5.6 and Remark 5.7]. The definition of a hom-hom PI ring given in [SZ, p. 1013] is equivalent to our definition of AS-regular. Note that a Krull domain of dimension 1 is a Dedekind domain (of global dimension 1). We refer to [SZ, WZ1, WZ2 for the definitions of Auslander regular and Cohen-Macaulay.

Lemma 5.3. Let $H$ be a noetherian regular affine PI Hopf algebra.

(a) $H$ is $A S$ regular, Auslander regular and Cohen-Macaulay and GKdim $H=$ gldim $H$.

(b) $H$ is a direct sum of prime rings of the same GK-dimension and the center of $H$ is a direct sum of Krull domains of the same GK-dimension.

(c) $H$ is finite over its center and each prime direct summand is equal to its trace ring. 
(d) Every clique of $H$ is finite and localizable.

(e) If $\operatorname{GKdim} H=1$, then the center of $H$ is a direct sum of Dedekind domains.

(f) Let $P_{0}$ be the minimal prime ideal of $H$ contained in $\operatorname{ker} \epsilon$ and let $H_{0}=$ $H / P_{0}$. Then the number of maximal ideals in the clique containing $k$ is bounded by the PI degree of $H_{0}$.

(g) $i o(H) \leq P I . \operatorname{deg}\left(H_{0}\right)<\infty$.

Proof. By [BG, Theorem 1.14] $H$ is AS-regular.

(a)-(e) follow from [SZ, Theorems 5.4 and 5.6]. We need to show (f) and (g).

(f) By (b) we have $H=\bigoplus_{i=0}^{s} H_{i}$ where each $H_{i}$ is a prime component of $H$ and $\bigoplus_{i>0} H_{i}$ is contained in $\operatorname{ker} \epsilon$. Then $P_{0}=\bigoplus_{i>0} H_{i}$. By (c) $H_{0}$ equals its trace ring. The assertion follows from [Bra, Theorem 8].

(g) The integral is only dependent on the homological property of $H_{0}$. By forgetting the coalgebra structure of $H$ we might assume that $H$ is prime. By (Cond1), $\operatorname{Ext}_{H}^{d}\left(\int^{r}, k\right) \neq 0$ where $d=\operatorname{GKdim} H$. By the proof of Proposition 2.5, $i o(H)$ is bounded by the number of primes in the clique containing $k$. The assertion follows from (f).

Definition 5.4. Let $H$ be a noetherian Hopf algebra with finite GK-dimension. Suppose $\pi: H \rightarrow H_{0}$ is a Hopf algebra quotient map. We say $H_{0}$ is the connected component of $H$ if

(a) GKdim $H_{0}=$ GKdim $H$.

(b) The following universal property holds: for every quotient Hopf algebra homomorphism $f: H \rightarrow H^{\prime}$ of the same GK-dimension, there is a unique Hopf algebra homomorphism $g: H^{\prime} \rightarrow H_{0}$ such that $g f=\pi$.

The connected component of $H$ is denoted by $H_{\text {conn }}$. We say $H$ is connected if $H$ is a connected component of itself.

A connected component of $H$ is unique if it exists. So it is safe to call $H_{\text {conn }}$ the connected component of $H$. If $H$ is prime, then it is connected. The converse is not clear to us, though we will show that this is true for regular Hopf algebras of GK-dimension one [Theorem 6.5. We hope to see that this is true for noetherian affine PI Hopf algebras.

Lemma 5.5. Let $H$ be a noetherian affine PI regular Hopf algebra and let $H^{\prime}$ be a quotient Hopf algebra of $H$. Suppose that GKdim $H=\operatorname{GKdim} H^{\prime}=d$.

(a) $H^{\prime}$ is regular. Further $\int_{H}^{l}=\int_{H^{\prime}}^{l}$ as right $H$-modules and $i o\left(H^{\prime}\right)=i o(H)$.

(b) $H^{\prime}$ is a direct summand of $H$.

(c) $H_{\text {conn }}$ exists.

Proof. By WZ1, Theorem 0.3], $H^{\prime}$ is projective over $H$ on both sides. Then we have, for all $i>d$ and all left $H^{\prime}$-modules $M$,

$\operatorname{Ext}_{H^{\prime}}^{i}\left(H_{H^{\prime}} k, H_{H^{\prime}} M\right)=\operatorname{Ext}_{H^{\prime}}^{i}\left(H^{\prime} \otimes_{H} k, H_{H^{\prime}} M\right) \cong \operatorname{Ext}_{H}^{i}\left(k, \operatorname{Hom}_{H^{\prime}}\left(H^{\prime}, M\right)\right)=0$.

Hence $k$ has finite projective dimension over $H^{\prime}$. By [BG, Corollary 1.4(c)], $H^{\prime}$ is regular. By Lemma 5.3(b), $H^{\prime}$ is a direct sum of prime rings of the same GKdimension. Thus $H^{\prime}$ is a factor ring of $H$ modulo some prime components. Hence $H \cong H^{\prime} \oplus A$ for some algebra $A$. Since the trivial $H$-module is also an $H^{\prime}$-module we have $\operatorname{Ext}_{H}^{d}(k, H) \cong \operatorname{Ext}_{H^{\prime}}^{d}\left(k, H^{\prime}\right)$. This shows that $\int_{H}^{l} \cong \int_{H^{\prime}}^{l}$ as right $H$-modules. Similarly, $\int_{H}^{r}=\int_{H^{\prime}}^{r}$. This implies that $\left(\int_{H}^{l}\right)^{\otimes p} \cong\left(\int_{H^{\prime}}^{l}\right)^{\otimes p}$ for all $p$ as right $H$ modules. The assertion of $i o(H)=i o\left(H^{\prime}\right)$ follows. 
Finally we need to prove (c), namely, to show the universal property in Definition 5.4. By Lemma 5.3(b), $H$ is a direct sum of prime rings of the same GK-dimension. So we can write it as $H=\bigoplus H_{i}$, as a decomposition of algebras. By the above proof, we see that every quotient Hopf algebra $H^{\prime}$ is the factor $\operatorname{ring} H / P$ where $P=\bigoplus_{j} H_{j}$, where $\{j\}$ is a subset of $\{i\}$. If $P$ and $Q$ are such Hopf ideals, then $P+Q$ is also a Hopf ideal. Hence $P+Q \neq H$ because both $P$ and $Q$ are contained in ker $\epsilon$. Therefore $P+Q=\oplus_{j} H_{j}$ is a direct summand of $H$. Let $M$ be the union of all such Hopf ideals. Then $H_{0}=H / M$ has the desired universal property and hence $H_{0}=H_{\text {conn }}$.

\section{Regular Hopf algebras of GK-Dimension 1}

The statement in Lemma 5.3 (b) asserting that $H$ is a direct sum of primes is analogous to the classical fact of algebraic groups: Suppose char $k=0$. Let $G$ be an algebraic group and let $G_{0}$ be the connected component of the identity of $G$. Then $G$ is a finite disjoint union of $g G_{0}$ for some $g \in G$ and there is a short exact sequence of algebraic groups

$$
0 \rightarrow G_{0} \rightarrow G \rightarrow G / G_{0} \rightarrow 0
$$

where $G / G_{0}$ is a finite group, which is called the discrete part of $G$. Dually, for a noetherian regular commutative Hopf algebra $H$, we have a short exact sequence of Hopf algebras

$$
0 \rightarrow H_{\text {dis }} \rightarrow H \rightarrow H_{\text {conn }} \rightarrow 0
$$

where $H_{d i s}$ is the maximal finite dimensional normal Hopf subalgebra of $H$ and where $H_{\text {conn }}$ is the quotient Hopf algebra $H /\left(H_{\text {dis }}\right)^{+} H$. The Hopf algebra $H_{\text {conn }}$ is a domain.

In this section we are aiming for a similar statement for noetherian regular noncommutative Hopf algebras of GK-dimension 1. Due to the noncommutativity of $H$ there are some extra structures of $H$ related to the integrals of $H$. For simplicity throughout this section

let $H$ be a noetherian affine PI regular Hopf $k$-algebra of $G K$-dimension 1.

In some parts we also assume that $k$ is algebraically closed as will be stated, but this assumption is not needed in the main result. Recall that $A^{*}$ means the $k$-linear dual of $A$.

Recall that the integral quotient of $H$, denoted by $H_{i q}$, is isomorphic to $H / J_{i q}$. When $i o(H)=n<\infty, J_{i q}=\bigcap_{p=0}^{n-1} \operatorname{ker}\left(\left(\Sigma^{r}\right)^{p}: H \rightarrow k\right)$ [Lemma 4.4], or equivalently, $J_{i q}=\bigcap_{p} \operatorname{l.ann}_{H}\left(\left(\int^{r}\right)^{\otimes p}\right)$. By Lemma 4.4 $H_{i q}^{\circ} \cong k \mathbb{Z}_{n}$. Of course if $k$ is algebraically closed and char $k \nmid n$, then $H_{i q} \cong k \mathbb{Z}_{n}$. The definitions of normal Hopf subalgebras, normal Hopf ideals and conormal homomorphisms are given in Mo, pp. 33-36].

Proposition 6.1. Let $J_{i q}=\bigcap_{p} \operatorname{lann}_{H}\left(\left(\int^{r}\right)^{\otimes p}\right)$ and $H_{i q}=H / J_{i q}$. Then the Hopf algebra homomorphism $H_{a b} \rightarrow H_{i q}$ is conormal.

We need a few lemmas to prove this proposition. First we need a reduction to the case when $k$ is algebraically closed.

Lemma 6.2. Let $F$ be a field extension of the base field $k$. Let $H_{F}=H \otimes F$.

(a) $H_{F}$ is a noetherian affine PI regular Hopf algebra of GK-dimension 1.

(b) $\int_{H_{F}}^{r}=\int^{r} \otimes F$ and $\int_{H_{F}}^{l}=\int^{l} \otimes F$. 
(c) $i o(H)=i o\left(H_{F}\right)$.

(d) $J_{F}:=\bigcap_{p}$ l.ann $_{H_{F}}\left(\left(\int_{H_{F}}^{r}\right)^{\otimes p}\right)=\bigcap_{p}$ l.ann $n_{H}\left(\left(\int^{r}\right)^{\otimes p}\right) \otimes F$. As a consequence, $J_{F}$ is a Hopf ideal of $H_{F}$ (if and only if $J_{i q}$ is a Hopf ideal of $H$ ).

(e) Proposition 6.1 holds for $H$ if and only if it holds for $H_{F}$.

Proof. (a) Field extension clearly preserves the following properties: "noetherian", "affine PI" and "GK-dimension 1". To prove that $H_{F}$ is regular, we only need to show that the trivial $H_{F}$-module $F$ has finite projective dimension. This follows from the fact that

$$
\operatorname{projdim}_{H_{F}} F=\operatorname{projdim}_{H \otimes F}(k \otimes F)=\operatorname{projdim}_{H} k=1
$$

when $H$ is noetherian.

(b), (c) Since $H$ is noetherian, by the Künneth formula,

$$
\int_{H_{F}}^{l}=\operatorname{Ext}_{H_{F}}^{1}\left(F, H_{F}\right)=\operatorname{Ext}_{H \otimes F}^{1}(k \otimes F, H \otimes F) \cong \operatorname{Ext}_{H}^{1}(k, H) \otimes F=\int^{l} \otimes F .
$$

Similarly, $\int_{H_{F}}^{r}=\int^{r} \otimes F$ and $i o\left(H_{F}\right)=i o(H)$.

(d) This is also clear from (b).

(e) This is true because of (b) and the fact the algebra $H / J_{i q}$ is isomorphic to a finite direct sum of $k$.

By the above lemma we only need to prove Proposition 6.1 for $H_{F}$ where $F$ is the algebraic closure of $k$. In other words we may assume $k$ to be algebraically closed. The following lemma is obvious because $H_{i q}=k$.

Lemma 6.3. If $H$ is unimodular, then Proposition 6.1 holds trivially.

Next we deal with the case when $i o(H) \neq 1$. Let $H^{\circ}$ be the dual Hopf algebra of $H$. For any ideal $P$ of $H$ of codimension one, let $\pi_{P}: H \rightarrow H / P \cong k$ denote the corresponding group-like element in $H^{\circ}$. Let $M_{P}$ be the left $H$-module $H / P$. We identify the ideal $P$ with the module $M_{P}$ when we talk about the cliques. Note that if $P$ and $Q$ are two ideals of $H$ of codimension one, then there is another ideal $R$ of $H$ of codimension one such that $M_{P} \otimes M_{Q}=M_{R}$ where the module structure on the tensor is defined via the coproduct of $H$. In this case it also implies $\pi_{P} \pi_{Q}=\pi_{R}$ in $H^{\circ}$. Let $l . a n n_{H}\left(\int^{r}\right)=P_{0}$. Then $\int^{r}=M_{P_{0}}$ and, for every $t,\left(\int^{r}\right)^{\otimes t}=\left(M_{P_{0}}\right)^{\otimes t}$ is corresponding to $\left(\Sigma^{r}\right)^{t}$ in $H^{\circ}$.

Lemma 6.4. Let $Q$ be an ideal of $H$ of codimension one.

(a) $M_{Q} \otimes \int^{r} \cong \int^{r} \otimes M_{Q}$.

(b) The clique containing $M_{Q}$ is $\left\{M_{Q} \otimes\left(\int^{r}\right)^{\otimes t} \mid t \geq 0\right\}$.

(c) The group $\left\{\left(\Sigma^{r}\right)^{t}\right\}_{t \geq 1}$ is a finite central subgroup of the group generated by $\left\{\pi_{P}\right\} \subset H^{\circ}$ where $P$ runs over all possible ideals of $H$ of codimension one.

Proof. First we prove that the clique containing $\operatorname{ker} \epsilon$ is the set of primes associated to $\left\{\left(\int^{r}\right)^{\otimes t} \mid t \geq 0\right\}$. By [BW, p. 324], for any two maximal ideals $P$ and $Q, Q \leadsto P$ if

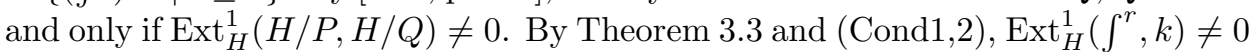
and $\operatorname{Ext}_{H}^{1}(T, k)=0$ for all other simple $H$-modules $T$. Hence the only left $H$-module linked to $\int^{r}$ is $k$. Since $M_{P} \otimes-$ and $-\otimes M_{P}$ are equivalences of the category of left $H$-modules, the only module linked to $M_{P} \otimes \int^{r}$ is $M_{P}$. For the same reason, the only module linked to $\int^{r} \otimes M_{P}$ is $M_{P}$. Therefore $M_{P} \otimes \int^{r}=\int^{r} \otimes M_{P}$. This proves (a). Let $P=P_{0}$. We obtain that $\left\{\left(\int^{r}\right)^{\otimes t} \mid t \geq 0\right\}$ is the clique containing 
k. Again using the functor $M_{P} \otimes-$, we obtain (b). By definition $\left\{\left(\int^{r}\right)^{\otimes t} \mid t \geq 0\right\}$ is a group that is finite by Lemma $5.3(\mathrm{~g})$.

(c) follows from (a).

Proof of Proposition 6.1. If $H$ is unimodular, see Lemma 6.3.

Now assume $i o(H) \neq 1$. By Lemma 6.2, we may assume that $k$ is algebraically closed. We will show the following.

(i) The abelianization $H_{a b}$ is finite dimensional and semisimple.

(ii) The group algebra $\bigoplus_{p} k\left(\Sigma^{r}\right)^{p}$ is a normal Hopf subalgebra of $\left(H_{a b}\right)^{\circ}$.

If $H_{a b}$ has GK-dimension 1, then, by Lemma [5.5(a), $i o(H)=i o\left(H_{a b}\right)=1$. This contradicts our hypothesis of $i o(H) \neq 1$. Hence $H_{a b}$ is finite dimensional. Since $k$ is algebraically closed of characteristic zero, $H_{a b}$ is a dual of a finite group algebra. This shows (i).

The dual Hopf algebra $\left(H_{a b}\right)^{\circ}$ is the group algebra $\oplus k \pi_{Q_{i}}$. By Lemma 6.4(c) $\bigoplus_{p} k\left(\Sigma^{r}\right)^{p}$ is a normal Hopf subalgebra of $\left(H_{a b}\right)^{\circ}$. This is (ii).

Note that (ii) is equivalent to the fact that the map $H_{a b} \rightarrow\left(\bigoplus_{p} k\left(\Sigma^{r}\right)^{p}\right)^{\circ}$ is conormal. Proposition 6.1 follows from the fact that $H_{i q}=\left(\bigoplus_{p} k\left(\Sigma^{r}\right)^{p}\right)^{\circ}$ [Lemma 4.4 .

Here is the main result of this section.

Theorem 6.5. Let $H=\bigoplus_{i=0}^{d} H_{i}$ be a decomposition of $H$ into prime components as in Lemma 5.3(b). Let $P_{0}$ be a minimal prime ideal contained in $\operatorname{ker} \epsilon$.

(a) $P_{0}$ is a Hopf ideal and $H / P_{0}$ is the connected component of $H$ in the sense of Definition [5.4, denoted by $H_{\text {conn }}$.

(b) The coinvariant subalgebra $H^{c o H_{c o n n}}$, denoted by $H_{\text {dis }}$, contains all finite dimensional normal Hopf subalgebras of $H$.

Remark 6.6. Both $H_{\text {conn }}$ and $H_{\text {dis }}$ are canonical objects associated to $H$. As is suggested by the commutative case and Theorem 6.5 it is natural to ask if $H_{d i s}$ is finite dimensional. It is also unclear to us if $H_{d i s}$ is a normal Hopf subalgebra of $H$. If these questions have positive answers we conjecture that there is a short exact sequence of Hopf algebras in the sense of [Sc, Definition 1.5]

$$
0 \rightarrow H_{\text {dis }} \rightarrow H \rightarrow H_{\text {conn }} \rightarrow 0
$$

where $H_{\text {conn }}=H /\left(H_{d i s}\right)^{+} H$. The conjectural description (E0.2.1) is an analog of E6.0.1) in the commutative case. This will be verified when $H$ is a group algebra [Proposition 8.2. Even if (E0.2.1) is false we can still ask if there is a Hopf algebra structure on the algebra $H_{d i s}$ such that $H$ is isomorphic to a crossed

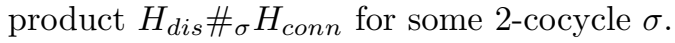

The point of Theorem6.5(a) is that $H_{\text {conn }}$ is a prime algebra which is not clear from Lemma 5.5. We need a few lemmas before proving Theorem 6.5.

Lemma 6.7. If there is a Hopf algebra quotient map $H \rightarrow H^{\prime}$ such that $H^{\prime}$ is prime and GKdim $H^{\prime}=1$, then $H^{\prime}$ is isomorphic to $H_{\text {conn }}$.

Proof. This follows from the existence of $H_{c o n n}$ in Lemma 5.5 and the universal property of $H_{\text {conn }}$ stated in Definition 5.4. 
Lemma 6.8. Assume the notation as in Theorem 6.5.

(a) If $K$ is a finite dimensional normal Hopf subalgebra of $H$, then $H / K^{+} H$ is infinite dimensional.

(b) If $H$ is prime, then there is no nontrivial finite dimensional normal Hopf subalgebra of $H$.

Proof. (a) [Brown] Let $K$ be a finite dimensional normal Hopf subalgebra of $H$. Then $H$ is a free left $K$-module [Sc, Theorem 2.1(2)], with a basis $\left\{h_{i} \mid i \in I\right\}$. Since $K$ is finite dimensional and $H$ is infinite dimensional, $I$ is infinite. Note that $H / K^{+} H$ is isomorphic to $\bigoplus_{i \in I} k h_{i}$. Thus $H / K^{+} H$ is infinite dimensional.

(b) Let $K$ be a finite dimensional normal Hopf subalgebra of $H$. If $K \neq k$, then $\bar{H}:=H / K^{+} H$ is finite dimensional because $H$ is prime of GK-dimension one, a contradiction to (a).

Lemma 6.9. Let $J_{i q}$ be as in Proposition 6.1. Then $\bigcap_{n} J_{i q}^{n}=P_{0}$.

Proof. First of all l.ann $(k)$ contains the minimal prime $P_{0}$. Since $H$ is a direct sum of primes and the ideals in the clique containing $k$ are related by the nonvanishing of $\operatorname{Ext}_{H}^{1}$, the ideals in the clique also contain $P_{0}$. Thus the assertion is equivalent to $\bigcap_{n} I^{n}=0$ where $I$ is the image of $J_{i q}$ in $H / P_{0}$. Replacing $H$ by $H / P_{0}$ (and forgetting the coalgebra structure of $H$ for a moment), it suffices to show that $\bigcap_{n} I^{n}=0$. By Lemma 5.3(d) $H$ is localizable at $I$ and the localization, denoted by $Q$, is semilocal. Since $H \rightarrow Q$ is injective, we only need to show that $\bigcap I^{\prime n}=0$ where $I^{\prime}=I Q$ is the Jacobson radical of $Q$. This is true for noetherian semilocal PI rings [MR, Corollary 6.4.15], and so we have proved the assertion.

Proof of Theorem 6.5. Since $J_{i q}$ is a Hopf ideal of $H$, by Lemma 4.7 $\bigcap_{n} J_{i q}^{n}$ is a Hopf ideal. By Lemma 6.9, $P_{0}$ is a Hopf ideal. Hence $H \rightarrow H / P_{0}$ is a Hopf algebra quotient with $H / P_{0}$ being prime and GK-dimension 1. By Lemma 6.7, $H / P_{0}$ is the connected component of $H$. Thus (a) is proved.

(b) Suppose $K$ is a finite dimensional normal Hopf subalgebra of $H$. Let $\bar{H}=$ $H / K^{+} H$. By [Mo, Proposition 3.4.3], $H^{c o \bar{H}}=K$. By (a) and Lemma 6.8(a), there is a canonical Hopf algebra quotient map from $\bar{H} \rightarrow H_{\text {conn } n}$. Hence $K \subset H_{d i s}$.

Corollary 6.10. Let $H$ be prime and $F$ be a field extension of $k$. Then $H_{F}:=$ $H \otimes F$ is prime.

Proof. By Lemma 6.9] if $H$ is prime, then $\bigcap_{n} J_{i q}^{n}=0$. By Lemma6.2(d), $\bigcap_{n} J_{F}^{n}=0$ where $J_{F}=\bigcap_{p}$ l.ann $H_{F}\left(\left(\int_{H_{F}}^{r}\right)^{\otimes p}\right)$. By Lemma 6.9, $H_{F}$ is prime.

\section{Regular Hopf algebras of GK-dimension 1: Prime case}

Throughout Section 7

let $H$ be a noetherian affine PI regular Hopf algebra of GK-dimension 1.

Here is the main result, which is a restatement of Theorem 0.2 .

Theorem 7.1. Let $H$ be prime.

(a) If $H$ is unimodular, then $H$ is commutative. If further $k$ is algebraically closed, then $H$ is isomorphic to either $k[x]$ or $k\left[x^{ \pm 1}\right]$.

(b) If $H$ is not unimodular with $i o(H)=n$, then the following holds:

(i) $n=P I \cdot \operatorname{deg}(H)$. 
(ii) The subalgebra of coinvariants $H_{c l}:=H^{c o H_{i q}}$ is a commutative domain.

(iii) $H_{c l}$ is affine and $H$ is a finite module over $H_{c l}$ on both sides.

In a previous version of this paper there was an extra hypothesis "char $k \nmid n$ " in Theorem 7.1(b)(iii). We thank Brown for providing us with a proof of the current version of the statement.

Remark 7.2. (a) The extra hypothesis of $k=\bar{k}$ in the second assertion of Theorem 7.1(a) is needed. When $k$ is not algebraically closed, there are other interesting Hopf algebras [Example 8.3.

(b) An immediate question after Theorem 7.1 is whether $H_{c l}$ is a Hopf subalgebra of $H$. This is not true as the next example shows. But we don't know if $H_{c l}$ is always equipped with a Hopf structure which may not be compatible with the Hopf structure of $H$. If this is the case, we can ask if there is a "twisted" short exact sequence

$$
0 \rightarrow H_{c l} \rightarrow H \rightarrow H_{i q} \rightarrow 0
$$

of Hopf algebras and if $H$ is isomorphic to a crossed product $H_{c l} \#_{\sigma} H_{i q}$.

(c) Theorem 7.1 fails for Hopf algebras of GK-dimension 2 (see Examples 2.9 and 8.5).

Example 7.3 (Continuation of Example 2.7). Let $H$ be the Hopf algebra defined in Example 2.7 for $n>1, m=1$. Since $m=1, H$ is prime. When char $k \nmid n$, then $H$ is regular. It is easy to see that $H_{i q}=H /(x) \cong k\langle g\rangle \cong k \mathbb{Z}_{n}$ and $H_{c l}$ is the subalgebra generated by $x$. Since $\Delta(x)=x \otimes 1+g \otimes x, H_{c l}$ is not a subcoalgebra of $H$. The ideal $\operatorname{ker}\left(H \rightarrow H_{i q}\right)$ is not normal in the sense of [Mo, Definition 3.4.5], or the homomorphism $H \rightarrow H_{i q}$ is not conormal. Let $H_{1}$ be the Hopf algebra $k[x]$ with $\Delta(x)=x \otimes 1+1 \otimes x$. Then $H_{1} \cong H_{c l}$ as algebras. It is easy to see that $H_{1}$ is an $H_{i q}$-Hopf module algebra and $H$ is a smash product $H_{1} \# k \mathbb{Z}_{n}$ [Example 2.7. This does suggest a "twisted" short exact sequence

$$
0 \rightarrow H_{1} \rightarrow H \rightarrow k \mathbb{Z}_{n} \rightarrow 0 .
$$

Also in this example, one has $H_{i q}=H_{a b}$, while the group algebra in Example 4.6 does not have this property.

Note that $\mathfrak{m}:=\operatorname{ker} \epsilon$ is idempotent. Hence $H_{\mathfrak{m}}^{\circ}$ is isomorphic to the trivial Hopf algebra $k$. On the other hand, $J_{i q}$ satisfies the condition $\bigcap_{n} J_{i q}^{n}=0$ [Lemma 6.9. Hence $H_{J_{i q}}^{\circ}$ is dense in $H^{*}$ (see the proof of [Mo, Proposition 9.2.10]).

We need a few lemmas before proving Theorem 7.1 A module is called uniserial if its submodules form a chain under inclusion. We will consider uniserial modules with a simple submodule. Let $T$ be a finite dimensional simple left $H$-module. Let $T^{\prime}$ denote the left $H$-module $\operatorname{Ext}_{H}^{1}(T, H)^{*}$. Since both $\operatorname{Ext}_{H}^{1}(-, H)$ and $(-)^{*}$ are equivalent functors on finite dimensional $H$-modules, $T^{\prime}$ is necessarily finite dimensional simple and $\operatorname{End}_{H}(T)=\operatorname{End}_{H}\left(T^{\prime}\right)$. Since $H$ is a Hopf algebra it follows from $(\mathrm{AS} 2)^{\prime}$ in Section 1 that $\operatorname{dim} T^{\prime}=\operatorname{dim} T$. Let $E(T)$ be the injective hull of $T$. Since $H$ is PI and $T$ is finite dimensional, $E(T)$ is locally finite.

Lemma 7.4. Let $T$ be a finite dimensional simple left $H$-module. Then $E(T)$ is an infinite dimensional uniserial module and the injective resolution of $T$ is

$$
0 \rightarrow T \rightarrow E(T) \rightarrow E\left(T_{0}\right) \rightarrow 0
$$


where $T_{0}$ is the unique finite dimensional simple $H$-module such that $T_{0}^{\prime} \cong T$. If $T$ is 1-dimensional, then $T \cong \int^{r} \otimes T_{0}$.

Proof. Recall that $H$ is regular of global dimension 1 . Then the minimal injective resolution of $T$ is

$$
0 \rightarrow T \rightarrow E(T) \rightarrow E(M) \rightarrow 0
$$

for some module $M$. Since $E(T)$ is locally finite, $E(M)$ is locally finite. By Theorem 3.3(b), there is a unique simple module $W$ such that $\operatorname{Ext}_{H}^{1}(W, T) \neq 0$ and that $W^{\prime}:=\operatorname{Ext}_{H}^{1}(W, H)^{*}$ is isomorphic to $T$. By notation $W=T_{0}$. By the definition of $\operatorname{Ext}_{H}^{1}(W, T), T_{0}$ is the unique simple module in the socle of $E(M)$. So we have the minimal injective resolution of $T$ :

$$
0 \rightarrow T \rightarrow E(T) \rightarrow E\left(T_{0}\right) \rightarrow 0 .
$$

Since $T$ and $T_{0}$ have no fundamental difference, $E(T)$ is infinite dimensional over $k$. If $N$ is a submodule of $E(T)$ of length 2, then $\operatorname{Ext}_{H}^{1}(N / T, T) \neq 0$. By what we just proved, $N / T \cong T_{0}$. Since $\operatorname{Ext}_{H}^{1}\left(T_{0}, T\right)=k, N$ is unique.

Next we use induction to show that a submodule of $E(T)$ is uniquely determined by its length. First we assume the length of a submodule $M \subset E(T)$, denoted by $l(M)$, is finite. If $l(M)=1$, then $M=T$, which is the socle of $E(T)$. Suppose the submodule of $E(T)$ of length $<s$ is unique. Now let $M$ be a submodule of length $s$. Let $N=M / T$ be the submodule in $E\left(T_{0}\right)$, which is of length $s-1$. Since $N$ is unique in $E\left(T_{0}\right), M$ is unique in $E(T)$. This takes care of the case of finite dimensional submodules $M$. Now assume that $M$ is infinite dimensional over $k$. Since $E(T)$ is locally finite, $M$ is not noetherian. One can easily construct an ascending sequence of submodules $M_{s}$ of length $s$. By what we just proved, the submodules $M_{s}$ are uniquely determined in $E(T)$. This means that $\left\{M_{s}\right\}$ is the complete set of finite dimensional submodules. Hence

$$
E(T)=\bigcup_{s} M_{s} \subseteq M \subseteq E(T) .
$$

If $T$ is a 1-dimensional left $H$-module, then $T_{0}=\int^{r} \otimes T$ since $\operatorname{Ext}_{H}^{1}\left(\int^{r} \otimes T, T\right) \cong$ $\operatorname{Ext}_{H}^{1}\left(\int^{r}, k\right)=k$.

We collect and fix some notations for the rest of this section.

Convention 7.5. Let $H$ be as in Theorem 7.1 and let $n=i o(H)$.

(a) For any simple left $H$-module $T$, let $E(T)$ be the injective hull of $T$ and let $E_{s}(T)$ be the unique submodule of $E(T)$ of length $s$. For every $s \geq 1$, let $E_{s}=\bigoplus_{i=0}^{n-1} E_{s}\left(\left(\int^{r}\right)^{\otimes i}\right)$. Let $E=\bigoplus_{i=0}^{n-1} E\left(\left(\int^{r}\right)^{\otimes i}\right)$.

(b) Let $\mathfrak{m}_{i}=$ l.ann $\left(\left(\int^{r}\right)^{\otimes i}\right)$ and let $J=J_{i q}=\bigcap_{i=0}^{n-1} \mathfrak{m}_{i}$. This is the defining ideal of $H_{i q}$. For every $s \geq 1$, let $H_{s}=H / J^{s}$. Let $\widehat{H}$ be the completion $\lim _{s} H_{s}$. Let gr $H$ be the associated graded ring $\bigoplus_{s} J^{s} / J^{s+1}$.

(c) Let $\Sigma_{i}$ be the quotient map from $H \rightarrow H / \mathfrak{m}_{i}$. We identify $\Sigma_{i}$ with $\left(\Sigma^{r}\right)^{i}$ in $H^{\circ}$. Let $\sigma_{i}$ be the algebra automorphism of $H$ determined by $\Sigma_{i}$. Then $\sigma_{i}=\left(\sigma^{r}\right)^{i}$ where $\sigma^{r}=\sigma_{1}$. The definitions of $\Sigma^{r}$ and $\sigma^{r}$ are given before Lemma 2.3. Let $G$ be the abelian group $\left\{\left(\Sigma^{r}\right)^{i}\right\}$ (which is isomorphic to $\left.\mathbb{Z}_{n}\right)$. Then $H_{i q}$ is isomorphic to $(k G)^{\circ}$. 
Lemma 7.6. Assume the notation as in Convention 7.5.

(a) $\sigma_{j}\left(\mathfrak{m}_{i}\right)=\mathfrak{m}_{i-j}$ for all $i, j$. Equivalently, $\sigma_{j}\left(\left(\int^{r}\right)^{\otimes i}\right)=\left(\int^{r}\right)^{\otimes(i-j)}$ as $\sigma_{j}$ acting on $H / J$.

(b) $H^{\text {coH }_{i q}}=H^{k G}$ where $G$ acts on $H$ via $\Sigma_{i} \rightarrow \sigma_{i}$.

(c) The canonical map $H \rightarrow \widehat{H}$ is injective and every automorphism $\sigma_{i}$ of $H$ extends to an automorphism of $\widehat{H}$, still denoted by $\sigma_{i}$.

(d) $E_{s}$ is the injective hull, as well as the projective cover, of $E_{1}$ as a left $H_{s^{-}}$ module.

(e) $H_{s} \cong E_{s}$ as left $H$-modules.

Proof. (a) This is equivalent to the fact $\pi_{i} \sigma_{j}=\pi_{i} \circ \pi_{j}=\pi_{i+j}$ for all $i, j$.

(b) This is true because the right $H_{i q}$-coaction on $H$ is equivalent to the right $H_{i q}^{\circ}$-action on $H$.

(c) The kernel of the map $H \rightarrow \lim _{s} H_{s}$ is $\bigcap_{i} J^{i}$ which is zero by Lemma 6.9. Hence $H \rightarrow \widehat{H}$ is injective.

By (a) $\sigma_{i}(J)=J$. Hence $\sigma_{i}\left(J^{t}\right)=J^{t}$ for all $t$. This means that $\sigma_{i}$ 's are automorphisms of $H_{s}$. Since $\widehat{H}=\varliminf_{s} H_{s}, \sigma_{i}$ 's extend to automorphisms of $\widehat{H}$ naturally.

(d) Since $E_{s}(T)$ is uniserial, so is $E_{s}(T) / J E_{s}(T)$. Thus $E_{s}(T) / J E_{s}(T)$ is simple. Thus shows that $\operatorname{Hom}_{H}\left(H_{s}, E\right)=E_{s}$. Hence $E_{s}$ is the injective hull of $E_{1}$ as an $H_{s}$-module. By the analogous statement for right modules, we see that the uniserial module $E_{s}^{*}$ is the injective hull of the right $H_{s}$-module $\left(E_{s} / E_{s-1}\right)^{*}$. By $k$-linear duality $(-)^{*}, E_{s}$ is the projective cover of the $H_{s}$-module $E_{1}$, since $E_{s} / E_{s-1} \cong E_{1}$.

(e) The assertion follows from (d) and the fact that $H_{s}$ is the projective cover of $H_{s} / J H_{s} \cong E_{1}$.

Proposition 7.7. Assume the notation as in Convention 7.5.

(a) $\widehat{H}$ is isomorphic to the matrix algebra

$$
H_{M}:=\left(\begin{array}{ccccc}
R & x R & \cdots & x^{n-2} R & x^{n-1} R \\
x^{n-1} R & R & \cdots & x^{n-3} R & x^{n-2} R \\
\cdots & \cdots & \cdots & \cdots & \cdots \\
x^{2} R & x^{3} R & \cdots & R & x R \\
x R & x^{2} R & \cdots & x^{n-1} R & R
\end{array}\right)
$$

where $R$ is a local ring $k\left[\left[x^{n}\right]\right]$.

(b) $\widehat{H}$ is a semilocal noetherian prime PI ring of global dimension and Krull dimension 1.

(c) Let $e_{i}=H / \mathfrak{m}_{i}$ for $i=1, \cdots, n$. Then $H / J=\widehat{H} / J(\widehat{H})=\bigoplus_{i} k e_{i}$ where $J(\widehat{H})$ is the Jacobson radical of $\widehat{H}$. The induced automorphism $\sigma_{j}$ maps $_{i}$ to $e_{i-j}$.

(d) gr $H$ is isomorphic to the algebra similar to $H_{M}$ where $R$ is $k\left[x^{n}\right]$ instead of $k\left[\left[x^{n}\right]\right]$. The induced automorphism $\sigma_{j}$ maps $e_{i}$ to $e_{i-j}$.

(e) The subring $\widehat{H}^{k G}$ is isomorphic to $k[[y]]$, whence it is a commutative local domain of global and Krull dimension 1.

(f) $H^{k G}$ is a commutative domain.

(g) $H^{k G}$ is affine and $H$ is a finite module over $H^{k G}$ on both sides. 
Proof. (a) For any map $f: M \rightarrow N$, we write $(m) f$ instead of $f(m)$ so that we don't need to take opposite ring when we have the canonical isomorphism $H \cong$ $\operatorname{End}_{H}\left({ }_{H} H\right)$.

Let $e_{i, i}$ be the identity map of $E\left(\left(\int^{r}\right)^{\otimes i}\right)$. Let $x_{0,1}$ be the fixed quotient morphism $E(k) \rightarrow E\left(\int^{r}\right)$ in the injective resolution of $k$. Then it induces a sequence of quotient morphism

$$
x_{i, i+1}: E\left(\left(\int^{r}\right)^{\otimes i}\right) \rightarrow E\left(\left(\int^{r}\right)^{\otimes(i+1)}\right)
$$

with the kernel $\left(\int^{r}\right)^{\otimes i}$. Clearly, $x_{i, i+1}=x_{i+n, i+1+n}$ for all $i$. We claim that

(i) $\operatorname{End}_{H}\left(E\left(\left(\int^{r}\right)^{\otimes i}\right)\right)=k\left[\left[y_{i}\right]\right]:=R_{i}$ where $y_{i}=x_{i, i+1} x_{i+1, i+2} \cdots x_{i+n-1, i+n}$ for all $i \in \mathbb{Z}_{n}$.

(ii) For $0<j-i<n, \operatorname{Hom}_{H}\left(E_{s}\left(\left(\int^{r}\right)^{\otimes i}\right), E_{s}\left(\left(\int^{r}\right)^{\otimes j}\right)\right)=x_{i, i+1} \cdots x_{j-1, j} R_{j}=$ $R_{i} x_{i, i+1} \cdots x_{j-1, j}$.

To prove (i) we note that $\operatorname{End}_{H}\left(E_{n}\left(\left(\int^{r}\right)^{\otimes i}\right)\right)=k$. By induction on $s$ one shows that $\operatorname{End}_{H}\left(E_{s n}\left(\left(\int^{r}\right)^{\otimes i}\right)\right)=k\left[y_{i}\right] /\left(y_{i}^{s}\right)$. Now we have

$$
\operatorname{End}_{H}\left(E\left(\left(\int^{r}\right)^{\otimes i}\right)\right)=\varliminf_{s} \operatorname{End}_{H}\left(E_{s n}\left(\left(\int^{r}\right)^{\otimes i}\right)\right)=k\left[\left[y_{i}\right]\right] .
$$

The proof of (ii) is similar.

Identifying $x_{i, i+1}$ with $e_{i, i+1} x$ inside the matrix algebra $M_{n}(k[[x]])$ where $\left\{e_{i, j}\right\}$ is the matrix unit, we have an isomorphism $\operatorname{End}_{H}(E) \cong H_{M}$. By Lemma 7.6(e), $H_{s} \cong E_{s}$ as left $H$-modules. So we can identify $E$ with $\varliminf_{s}\left(H_{s}\right)^{*}$. Hence

$$
\widehat{H}=\lim _{s} H_{s}=\lim _{s} \operatorname{End}_{H}\left(H_{s}\right) \cong \lim _{\longleftarrow} \operatorname{End}_{H}\left(E_{s}\right)=\operatorname{End}_{H}(E) .
$$

Therefore (a) is proved.

(b) This is clear.

(c) This follows from Lemma 7.6(a) and the fact $H / J=\widehat{H} / J(\widehat{H})$.

(d) Since $\operatorname{gr} H=\operatorname{gr} \widehat{H} \cong \operatorname{gr} H_{M}$. This is clear from the description of (a). Equivalently, in the notations in the proof of (a), gr $H$ is isomorphic to

$$
k\left\langle e_{0,0}, \cdots, e_{n-1, n-1}, x_{0,1}, x_{1,2}, \cdots, x_{n-1,0}\right\rangle /(\text { rels })
$$

where the ideal (rels) is generated by the relations $1=\sum_{i=0}^{n-1} e_{i, i}, e_{i, i} x_{i, k}=x_{i, k}=$ $x_{i, k} e_{k, k}$, and $0=e_{i, i} x_{j, k}=x_{k, j} e_{i, i}=x_{i, j} x_{k, l}$ if $j \neq k$ in $\mathbb{Z}_{n}$.

Since $\sigma_{j}$ maps $J$ to $J, \sigma_{j}$ induces an automorphism of gr $H$. Therefore $k G \cong$ $\bigoplus_{i} k \sigma_{j}$ acts on gr $H$. The second assertion follows from (c).

(e) First we compute the invariant subring ( $\operatorname{gr} H)^{k G}$ of gr $H$. By (d), $\sigma:=\sigma_{-1}$ maps $e_{i, i}$ to $e_{i+1, i+1}$. Using the equation

$$
\sigma\left(x_{i, i+1}\right)=\sigma\left(e_{i, i} x_{i, i+1} e_{i+1, i+1}\right)=e_{i+1, i+1} \sigma\left(x_{i, i+1}\right) e_{i+2, i+2}
$$

one sees that $\sigma\left(x_{i, i+1}\right)=w_{i} x_{i+1, i+2}$ for some $0 \neq w_{i} \in k$. Since $\sigma^{n}$ is the identity, we obtain that $w_{0} \cdots w_{n-1}=1$. Modifying $x_{i, i+1}$ by scalars, we may assume that $w_{i}=1$ for all $i$. Now $\sigma$ maps $e_{i, i} \rightarrow e_{i+1, i+1}$ and $x_{i, i+1} \rightarrow x_{i+1, i+2}$ for all $i \in \mathbb{Z}_{n}$. Now it is straightforward to compute that $(\operatorname{gr} H)^{k G}=k[x]$ where $x=\sum_{i=0}^{n-1} x_{i, i+1}$.

We now study the subring $(\widehat{H})^{k G}$. Let $y=\sum_{i=0}^{n-1} \sigma^{i}\left(x_{i, i+1}\right)$. Then gr $y=x$ because gr $y$ is a $\sigma$ invariant and in gr $H$ one has $\sum_{i=0}^{n-1} \sigma^{i}\left(x_{i, i+1}\right)=x$. We will show that $(\widehat{H})^{k G}=k[[y]]$. Define the degree of $f \in \widehat{H}$ to be the degree $\operatorname{gr} f$ in gr $H$. We claim that for every element $f$ in $(\widehat{H})^{k G}$, there is a $w \in k$ such that $\operatorname{deg}\left(f-w y^{p}\right)>p$ where $p=\operatorname{deg} f$. This is true because $\operatorname{gr} f$ is in $(\operatorname{gr} H)^{k G}$ and hence gr $f$ and $\operatorname{gr} y^{p}$ are in the same vector space $k x^{p}$. So we proved the claim. 
By induction and the claim, for any element $f \in \widehat{H}^{k G}$, there is a sequence of elements $w_{i} \in k$ such that $\operatorname{deg}\left(f-\sum_{i=0}^{t} w_{i} y^{i}\right)>t$ for all $t$. Since $\widehat{H}$ is complete $f=\sum_{i=0}^{\infty} w_{i} y^{i} \in k[[y]]$.

(f) Since $H \subset \widehat{H}$ we have $H^{k G} \subset(\widehat{H})^{k G}$. By (e) $H^{k G}$ is a commutative domain.

(g) [Brown] Let $Z$ be the center of $H$. Since $H$ is affine prime of GK-dimension one, $Z$ is an affine commutative domain and $H$ is a finite module over $Z$ on both sides [SSW. Since $G$ is a finite group of automorphism of $H$, it is a group of automorphisms of $Z$. Since $Z$ is affine, the invariant subring $Z^{G}$ is affine and $Z$ is a finite module over $Z^{G}$ by the Hilbert-Noether theorem [Be, Theorem 1.3.1]. Hence $H$ is a finite module over $Z^{G}$. Since $H^{k G}$ is a subring of $H$ containing $Z^{G}, H^{k G}$ is a finite module over an affine commutative subring $Z^{G}$. Therefore $H^{k G}$ is affine and $H$ is a finite module over $H^{k G}$ on both sides.

As a consequence, $H^{k G}$ has GK-dimension and Krull dimension 1.

Proof of Theorem 7.1. (a) If $H$ is unimodular, then, by Proposition 7.7, $H$ is a subring of $\widehat{H}=M_{1}(k[[x]])$. Hence $H$ is commutative.

For the second assertion we assume that $k$ is algebraically closed. The algebraic group associated to $H$ is connected, linear (i.e., affine) and of dimension 1. By [Sp, Theorem 2.6.6], any connected linear algebraic group of dimension 1 is isomorphic to either $\mathbb{G}_{a}:=(k,+)$ or $\mathbb{G}_{m}:=(k-\{0\}, \times)$. Thus $H$ is isomorphic to either $k[x]$ or $k\left[x^{ \pm 1}\right]$.

(b) Suppose $n=i o(H)>1$. By Proposition 7.7(a), the PI degree of $\widehat{H}$ is no more than $n$. Hence the PI degree of $H$ is no more than $n$. Combining with Lemma $5.3(\mathrm{~g})$, io $(H)=$ PI.deg $(H)$. This proves (i). The rest of (ii), (iii) follows from Proposition 7.7 (f), (g).

Corollary 7.8. Let $H$ be as in Theorem 7.1. Suppose $k$ is algebraically closed. The following are equivalent:

(a) $H$ is a domain.

(b) $H$ is commutative.

(c) $\epsilon\left(\int^{l}\right) \neq 0$ where $\epsilon$ is defined in (E3.0.1) (for $d=1$ ).

(d) $H$ is unimodular.

(e) $\operatorname{Ext}_{H}^{1}(k, k) \neq 0$.

(f) $H$ is isomorphic to either $k[x]$ or $k\left[x^{ \pm 1}\right]$.

Proof. (a) $\Rightarrow$ (b) Let $Q$ be the ring of fractions of $H$. Hence $Q$ is a division algebra of GK-dimension 1. We claim that every division algebra of GK-dimension 1 is commutative, which implies (b). It is clear that $Q$ is a direct union of finitely generated division algebras. We may assume that both $Q$ and its center, say $K$, are finitely generated as division algebras. Since the transcendence degree of $K$ is 1 and the base field $k$ is algebraically closed, by Tsen's theorem [Co, P. 374], the Brauer group of $K$ is trivial, whence $Q=K$. Therefore $Q$ is commutative.

(b) $\Rightarrow$ (c) Since $H$ is commutative, $\int^{r}=\int^{l}=k$, (c) follows from (Cond1) since $H$ is regular.

(c) $\Rightarrow$ (d) This is Lemma 3.1

(d) $\Rightarrow(\mathrm{e}) \operatorname{Ext}_{H}^{1}(k, k) \cong \operatorname{Ext}_{H}^{1}\left(\int^{r}, k\right) \cong k$.

(e) $\Rightarrow$ (c) By (Cond3) for $T=k$, we see that $\epsilon\left(\int^{l}\right) \neq 0$.

(d) $\Rightarrow$ (f) This is Theorem 7.1(a).

(f) $\Rightarrow$ (a) Trivial. 
Lemma 7.9. Let $H$ be a noetherian regular affine Hopf algebra of GK-dimension 1. Let $W$ and $T$ be left simple $H$-modules in the same clique. Then $\operatorname{dim} W=\operatorname{dim} T$, $\operatorname{End}_{H}(W) \cong \operatorname{End}_{H}(T)$ and PI.deg $\left(H / l \cdot \operatorname{ann}_{H}(W)\right)=P I \cdot d e g\left(H / l \cdot \operatorname{ann}_{H}(T)\right)$.

Proof. First of all the global dimension of $H$ will be 1 [Lemma 5.3(a)]. By Theorem 3.3. for every given simple module $S$, there is only one simple module $V=S^{\prime}$ such that $\operatorname{Ext}_{H}^{1}(S, V) \neq 0$. In this case we write $V \leadsto S$. This uniqueness property implies there is a unique sequence of simple modules $\left\{T_{0}, T_{1}, \cdots, T_{n}\right\}$ such that either

$$
T=T_{0} \leadsto T_{1} \leadsto \cdots \leadsto T_{n}=W
$$

or

$$
W=T_{0} \leadsto T_{1} \leadsto \cdots \leadsto T_{n}=T .
$$

By induction on $n$ and the left-right symmetry we may assume that $T \leadsto W$. In this case $T=W^{\prime}=\operatorname{Ext}_{H}^{1}(W, H)^{*}$. Since $H$ is AS-Gorenstein, $\operatorname{Ext}_{H}^{1}(-, H)$ is an equivalent functor $\operatorname{Mod}_{f d^{-}} H \rightarrow \operatorname{Mod}_{f d^{-}} H^{o p}$. Hence we have $\operatorname{End}_{H}\left(W^{\prime}\right)=\operatorname{End}_{H}(W)$. By $(\mathrm{AS} 2)^{\prime}$ in Section $1, \operatorname{dim} W^{\prime}=\operatorname{dim} W$. Finally PI.deg $\left(H / l \cdot a n n_{H}(W)\right.$ is determined by $\operatorname{dim} W$ and $\operatorname{dim} \operatorname{End}_{H}(W)$.

Proof of Corollary 0.3, By [SZ, Remark 5.7(ii)], two maximal ideals $I$ and $J$ of $H$ are in the same clique if and only if $I \cap Z(H)=J \cap Z(H)$ where $Z(H)$ is the center of $H$. Let $I=\operatorname{l.ann}_{H}(M)$. By [Bra, Theorem 8] we have

$$
P I . \operatorname{deg}(H)=\sum_{J} c_{J} P I . \operatorname{deg}(H / J),
$$

for some integers $c_{J}$, where $J$ runs over all maximal ideals in the clique containing $I$. Let $M_{J}$ be the simple left $H$-modules corresponding to $J$. By Lemma $7.9 \operatorname{dim} M_{J}=$ $\operatorname{dim} M$ and PI.deg $(H / I)=P I \cdot \operatorname{deg}(H / J)$ for all $J$. Since $k$ is algebraically closed, $P I . \operatorname{deg}(H / I)=\operatorname{dim} M$. Then (E7.10.1) becomes

$$
P I . \operatorname{deg}(H)=\left(\sum_{J} c_{J}\right) \operatorname{dim} M .
$$

The assertion follows by Theorem 0.2 (a).

Note that we can show that coefficients $c_{J}$ in (E7.10.1) are 1, but the proof is omitted.

\section{Group algebras}

There is nothing new in this section. The reason we include this short section is to show how the integral and the integral order can be related to the structures of groups. The following lemma is well-known.

Lemma 8.1. Let $G$ be a group and $H^{\prime}$ be a Hopf algebra. Suppose $f: k G \rightarrow H^{\prime}$ is a surjective Hopf algebra homomorphism. Then

(a) $H^{\prime}=k G^{\prime}$, where $G^{\prime}=f(G)$, and $f$ is induced by the group homomorphism $\left.f\right|_{G}: G \rightarrow G^{\prime}$

(b) $k G_{0}=(k G)^{\text {co } k G^{\prime}}$, where $G_{0}$ is the kernel of $\left.f\right|_{G}$.

Let $G$ be a finitely generated group. We say $G$ has linear growth if (a) $G$ is infinite and (b) there is a generating set $T \subset G$ with $1 \in T$ and $T^{-1}=T$ such that $\left|T^{n}\right| \leq c n$ for some constant $c>0$. By $\mathrm{WV}$, if $G$ is sub-quadratic (meaning that $\left|T^{n}\right|-\left|T^{n-1}\right|<n$ for some $n$ ), then $G$ has linear growth and contains a subgroup 
$\mathbb{Z}$ of finite index. As a consequence, $k G$ is noetherian of GK-dimension 1 . Such a group $G$ is said to have two ends [IS, p. 100]. Conversely, if $k G$ is a noetherian Hopf algebra of GK-dimension 1, then $G$ is finitely generated with linear growth. By [Pas, Theorem 3.13 in Chapter 10] $k G$ is regular if $k$ is of characteristic zero (this can be weakened, dependent on the order of finite subgroups of $G$ ).

The conjectural descriptions $(\mathrm{E} 0.2 .1)$ and $(\mathrm{E} 0.2 .2)$ are verified for group rings. The following result is basically known [S, St, and we remark that it gives the short exact sequences (E0.2.1) and (E0.2.2).

Proposition 8.2. Let $G$ be a finitely generated group with linear growth.

(a) There is a finite normal subgroup $G_{d i s} \subset G$ such that it contains all finite normal subgroups of $G$. Also, there is a short exact sequence

$$
1 \rightarrow G_{d i s} \rightarrow G \rightarrow G_{\text {conn }} \rightarrow 1
$$

where $G_{\text {conn }}=G / G_{\text {dis }}$ contains no nontrivial finite normal subgroup.

(b) The connected component $G_{\text {conn }}$ is isomorphic to either $\mathbb{Z}$ or $\mathbb{D}$ [Example 4.6]. In either case, $G_{\text {conn }}$ fits into a short exact sequence

$$
1 \rightarrow \mathbb{Z} \rightarrow G_{\text {conn }} \rightarrow G_{i q} \rightarrow 1
$$

where $G_{i q}$ is either $\{1\}$ or $\mathbb{Z}_{2}$.

(c) $i o(k G)=1$ or 2 , corresponding to the two cases in (b).

It follows from Proposition 8.2(a), (b) that (E0.2.1) and (E0.2.2) hold for $H=k G$ when $k G$ is affine regular of GK-dimension 1.

Proof of Proposition 8.2. (a) We take $k=\mathbb{C}$ for simplicity and let $H=k G$. Then $k G$ is a regular noetherian affine PI Hopf algebra of GK-dimension 1. By Theorem 6.5. there is a surjective Hopf algebra homomorphism $f: k G \rightarrow H_{\text {coon }}$ with $H_{\text {conn }}$ being prime. By Lemma 8.1(a), $H_{c o n n}$ is a group algebra, denoted by $k G_{c o n n}$, and $G_{c o n n}$ is a quotient group of $G$. Since $k G_{c o n n}$ is prime, $G_{c o n n}$ contains no nontrivial finite normal subgroup. We obtain the short exact sequence by letting $G_{d i s}=\operatorname{ker}\left(G \rightarrow G_{c o n n}\right)$. Since $G_{c o n n}$ is infinite and $G$ has linear growth, $G_{d i s}$ is finite. By Theorem 6.5(b), $G_{d i s}$ contains all finite normal subgroups of $G$.

(b) Now assume that $k G$ is prime. If $k G$ is commutative, then $G$ is abelian and it must be $\mathbb{Z}$ by the decomposition of finitely generated abelian groups. Now assume $k G$ is not commutative. By Theorem 7.1, there is a Hopf algebra homomorphism $k G \rightarrow H_{i q}$ such that $(k G)^{c o} H_{i q}$ is a commutative domain. Note that $H_{i q}=\left(k \mathbb{Z}_{n}\right)^{\circ} \cong k \mathbb{Z}_{n}$ where $n=i o(k G)$. By Lemma 8.1. $(k G)^{c o} H_{i q}$ is a group algebra of GK-dimension 1 , hence $(k G)^{c o} H_{i q}=k \mathbb{Z}$. So we have a short exact sequence

$$
1 \rightarrow \mathbb{Z} \rightarrow G \rightarrow \mathbb{Z}_{n} \rightarrow 1,
$$

which gives rise to the canonical map $k G \rightarrow(k G)_{i q}$. Let $K$ be a maximal normal abelian subgroup of $G$ containing $\mathbb{Z}$ in (E8.2.1). So we have a short exact sequence

$$
1 \rightarrow K \rightarrow G \rightarrow \mathbb{Z}_{m} \rightarrow 1
$$

for $1<m \leq n$. Since $G$ does not contain a proper finite normal subgroup, it is easy to show that $K$ does not contain a proper finite subgroup. Thus $K \cong \mathbb{Z}$. If the action of $\mathbb{Z}_{m}$ on $K$ is not faithful, then it produces a larger normal abelian group containing $K$, a contradiction. Therefore the action of $\mathbb{Z}_{m}$ on $K$ is faithful. The 
only nontrivial action of $K \cong \mathbb{Z}$ is $n \rightarrow-n$. Thus $m=2$. In this case $G \cong \mathbb{D}$, which is described in Example 4.6.

(c) Follows from (b) and Example 4.6. Note that the assertion holds for any field $k$ such that $k G$ is regular.

The following example is provided by Stafford.

Example 8.3. Let $k$ be a field of $\operatorname{char} k \neq 2$. Suppose that $i:=\sqrt{-1} \notin k$. One might assume $k=\mathbb{R}$ for simplicity. Let $H$ be the algebra $k[x, y] /\left(x^{2}+y^{2}-1\right)$. This is a Hopf algebra because it is the coordinate ring of the unit circle, which is an algebraic group at least when $k=\mathbb{R}$. The coalgebra structure of $H$ is determined by

$$
\Delta(x)=x \otimes x-y \otimes y, \quad \Delta(y)=x \otimes y+y \otimes x
$$

and the counit and the antipode are determined by

$$
\epsilon(x)=1, \epsilon(y)=0 \quad \text { and } \quad S(x)=x, S(y)=-y .
$$

Since $i \notin k$, this Hopf algebra is not isomorphic to the group algebra $k \mathbb{Z}\left(\cong k\left[t, t^{-1}\right]\right)$ over the base field $k$. Let $F$ be any field extension of $k$ such that $i \in F$, or one can take $F=\bar{k}$. Then $H \otimes_{k} F$ is isomorphic to $F\left[z, z^{-1}\right] \cong F \mathbb{Z}$ where $z=x+i y$ and $z^{-1}=x-i y$. So there are two nonisomorphic Hopf algebras, namely, $k \mathbb{Z}$ and $H$, such that their field extensions are isomorphic as Hopf algebras.

A Hopf quotient of $H$ was studied by Greither and Pareigis GP. Let $H_{1}=$ $H /(x y)$. Then $H_{1}$ is a finite dimensional Hopf algebra not isomorphic to a group algebra, and $H_{1} \otimes_{k} F$ is isomorphic to a group algebra $F\left(\mathbb{Z}_{2} \times \mathbb{Z}_{2}\right)$. This Hopf algebra is called the circle Hopf algebra or the trigonometric Hopf algebra (see also Par] and [Mo, pp.125-6]).

An easy extension can be made without restriction on char $k$ as follows. Let $\xi \in k$ such that $\sqrt{-\xi} \notin k$. Let $H_{\xi}$ be the algebra $k[x, y] /\left(x^{2}+\xi y^{2}-1\right)$ with other operations

$$
\Delta(x)=x \otimes x-\xi y \otimes y, \quad \Delta(y)=x \otimes y+y \otimes x
$$

and

$$
\epsilon(x)=1, \epsilon(y)=0 \quad \text { and } \quad S(x)=x, S(y)=-y .
$$

Then $H_{\xi}$ is a Hopf algebra.

It is easy to check that if $K$ is a Hopf algebra such that $K \otimes_{k} F$ is isomorphic to the Hopf algebra $F[z]$ for some field extension $F \supset k$, then $H \cong k[x]$.

One can also construct a similar Hopf algebra $H^{\prime}$ such that $H^{\prime} \neq k \mathbb{D}$ (see Example 4.6 for the definition of $\mathbb{D}$ ); but for some field extension $F, H^{\prime} \otimes_{k} F \cong F \mathbb{D} \cong$ $k \mathbb{D} \otimes_{k} F$.

Question 8.4. When $k$ is algebraically closed, the group algebras in Proposition 8.2 and the Hopf algebras in Example 2.7 (for $m=1$ ) are the only examples of affine prime regular Hopf algebras of GK-dimension 1 we know so far. Are there others?

When $k$ is not algebraically closed, there are some others [Example 8.3. What can we expect in this case? 
Finally we give an example that shows that Theorem 0.2 fails for group algebras of GK-dimension 2.

Example 8.5. Let $G$ be the group $\left\langle g, x, y \mid g^{2}=1, g x g=x^{-1}, g y g=y^{-1}, x y=y x\right\rangle$. It's easy to see that the subgroup $\langle y\rangle$ is normal and $G /\langle y\rangle$ is isomorphic to the group $\mathbb{D}$ defined in Example 4.6 .

By using the relations between $g, x$ and $y$, we see that

$$
g(y-1)=\left(y^{-1}-1\right) g=(y-1)\left(-y^{-1} g\right) \quad \text { and } \quad x(y-1)=(y-1) x .
$$

Hence $y-1$ is a normal element in the group algebra $k G$ and $k G /(y-1) \cong k \mathbb{D}$. By Example 4.6 the right integral of $k \mathbb{D}$ is isomorphic to $k \mathbb{D} /(x-1, g+1)$. By Lemma 2.6(b), the right integral of $k G$ is isomorphic to the trivial $k G$-module $k$. Hence the integral order is 1 . This shows the first property listed below. The other properties are clear.

(a) $i o(k G)=1$.

(b) $k G$ is a prime ring.

(c) $k G$ has global dimension and GK-dimension 2 .

(d) $k G$ is not a domain.

(e) $k G$ is a PI ring of PI degree 2 , hence not commutative.

Therefore all statements in Theorem 0.2 fail for this group algebra.

\section{ACKNOWLEDGMENTS}

D.-M. Lu was supported by the NSFC (project 10571152). Q.-S. Wu was supported by the NSFC (key project 10331030) and by STCSM (03JC14013) and also supported by the Cultivation Fund of the Key Scientific and Technical Innovation Project, Ministry of Education of China (NO 704004). J.J. Zhang was supported by NSF grant DMS-0245420 (USA) and Leverhulme Research Interchange Grant F/00158/X (UK). The authors thank Jacques Alev, Ken Brown, Ken Goodearl, Tom Lenagan, Martin Lorenz, Monty McGovern, Susan Montgomery, John Palmieri, Paul Smith and Toby Stafford for many useful discussions and valuable comments. In particular the authors thank Toby Stafford for providing Example 8.3 and Ken Brown for the proofs of Lemma 6.8(a) and Theorem 7.1(b)(iii).

\section{Note ADDED IN PROOF (APRIL 2007)}

Recently, G.-X. Liu constructed a new affine noetherian prime regular Hopf algebra of GK-dimension one [Liu. Hence he answered Question 8.4 and the question stated in Remark 7.2(b).

\section{REFERENCES}

[ASZ] K. Ajitabh, S.P. Smith and J.J. Zhang, Auslander-Gorenstein rings, Comm. Algebra 26 (1998), no. 7, 2159-2180. MR.1626582(99g:16010)

[Be] D.J. Benson, Polynomial invariants of finite groups, London Mathematical Society Lecture Note Series, 190. Cambridge University Press, Cambridge, 1993. MR 1249931 (94j:13003)

[Bra] A. Braun, An additivity principle for p.i. rings. J. Algebra 96 (1985), no. 2, 433-441. MR0810539 (87f:16011) 
[BW] A. Braun and R.B. Warfield Jr., Symmetry and localization in Noetherian prime PI rings. J. Algebra 118 (1988), no. 2, 322-335. MR0969675 (89k:16031)

[Bro] K. A. Brown, Representation theory of Noetherian Hopf algebras satisfying a polynomial identity, Trends in the representation theory of finite-dimensional algebras (Seattle, WA, 1997), 49-79, Contemp. Math., 229, AMS, Providence, RI, 1998. MR.1676211|(99m:16056)

[BG] K. A. Brown and K. R. Goodearl, Homological aspects of Noetherian PI Hopf algebras of irreducible modules and maximal dimension, J. Algebra 198 (1997), 240-265. MR1482982 (99c:16036)

[Co] P.M. Cohn, Algebra. Vol. 2. With errata to Vol. I. John Wiley \& Sons, London-New YorkSydney, 1977. MR0530404 (58:26625)

[GW] K.R. Goodearl and R.B. Warfield Jr., "An introduction to noncommutative Noetherian rings", Second edition. London Mathematical Society Student Texts, 61. Cambridge University Press, Cambridge, 2004. MR2080008 (2005b:16001)

[GP] C. Greither and B. Pareigis, Hopf Galois theory for separable field extensions, J. Algebra 106 (1987), no. 1, 239-258. MR0878476 (88i:12006)

[IS] W. Imrich and N. Seifter, A bound for groups of linear growth, Arch. Math. (Basel) 48 (1987), no. 2, 100-104. MR0878419 (88e:20035)

[LR1] R.G. Larson and D.E. Radford, Finite-dimensional cosemisimple Hopf algebras in characteristic 0 are semisimple, J. Algebra 117 (1988), no. 2, 267-289. MR.0957441 (89k:16016)

[LR2] R.G. Larson and D.E. Radford, Semisimple cosemisimple Hopf algebras, Amer. J. Math. 110 (1988), no. 1, 187-195. MR0926744 (89a:16011)

[LS] R.G. Larson and M. Sweedler, An associative orthogonal bilinear form for Hopf algebras, Amer. J. Math. 91 (1969) 75-94. MR0240169 (39:1523)

[Liu] G.-X. Liu, On Noetherian affine prime regular Hopf algebras of Gelfand-Kirillov dimension 1, Proc. Amer. Math. Soc. (to appear).

[LL] M.E. Lorenz and M. Lorenz, On crossed products of Hopf algebras, Proc. Amer. Math. Soc. 123 (1995), no. 1, 33-38. MR1227522(95c:16014)

[MR] J. C. McConnell and J. C . Robson, "Noncommutative Noetherian Rings," Wiley, Chichester, 1987. MR0934572 (89j:16023)

[Mo] S. Montgomery, "Hopf algebras and their actions on rings", CBMS Regional Conference Series in Mathematics, 82, Providence, RI, 1993. MR.1243637(94i:16019)

[Par] B. Pareigis, Forms of Hopf algebras and Galois theory, Topics in algebra, Part 1 (Warsaw, 1988), 75-93, Banach Center Publ., 26, Part 1, PWN, Warsaw, 1990 MR.1171227 (93f:16038)

[Pas] D.S. Passman, The algebraic structure of group rings, Reprint of the 1977 original. Robert E. Krieger Publishing Co., Inc., Melbourne, FL, 1985. MR0798076 (86j:16001)

[Ro] J.J. Rotman, An introduction to homological algebra, Pure and Applied Mathematics, 85. Academic Press, Inc. New York-London, 1979. MR0538169 (80k:18001)

[Sc] H.-J. Schneider, Some remarks on exact sequences of quantum groups. Comm. Algebra 21 (1993), no. 9, 3337-3357. MR1228767 (94e:17026)

[SSW] L. W. Small, J.T. Stafford and R. B. Warfield Jr., Affine algebras of Gelfand-Kirillov dimension one are PI, Math. Proc. Cambridge Philos. Soc. 97 (1985), no. 3, 407-414. MR0778674 (86g:16025)

[Sp] T.A. Springer, Linear algebraic groups, 2nd edition. Birkhäuser Boston, Inc., Boston, MA, 1998. MR 1642713 (99h:20075)

[St] J. Stallings, Group theory and three-dimensional manifolds, Yale University Press, New Haven, Conn.-London, 1971. MR 0415622(54:3705)

[SZ] J. T. Stafford and J. J. Zhang, Homological properties of (graded) Noetherian PI rings, J. Algebra 168 (1994), no. 3, 988-1026. MR1293638 (95h:16030)

[Ta] E.J. Taft, The order of the antipode of finite-dimensional Hopf algebra, Proc. Nat. Acad. Sci. U.S.A. 68 (1971), 2631-2633. MR0286868(44:4075)

[WV] A.J. Wilkie and L. van den Dries, An effective bound for groups of linear growth, Arch. Math. (Basel) 42 (1984), no. 5, 391-396. MR0756689 (85k:20107)

[WZ1] Q.-S. Wu and J.J. Zhang, Noetherian PI Hopf algebras are Gorenstein, Trans. Amer. Math. Soc. 355 (2003), no. 3, 1043-1066. MR.1938745 (2003m:16056)

[WZ2] Q.-S. Wu and J.J. Zhang, Regularity of Involutory PI Hopf Algebras, J. Algebra 256 (2002), no. 2, 599-610. MR1939124 (2004g:16042) 
[WZ3] Q.-S. Wu and J.J. Zhang, Homological identities for noncommutative rings, J. Algebra, 242 (2001), 516-535. MR.1848957 (2002k:16011)

[YZ] A. Yekutieli and J.J. Zhang, Residual complex over noncommutative rings, J. Algebra 259 (2003), no. 2, 451-493. MR1955528 (2004a:16010)

Department of Mathematics, Zhejiang University, Hangzhou 310027, People's RepubLIC OF CHINA

E-mail address: dmlu@zju.edu.cn

Institute of Mathematics, Fudan University, Shanghai, 200433, People's Republic of CHINA

E-mail address: qswu@fudan.edu.cn

Department of Mathematics, Box 354350, University of Washington, Seattle, WashINGTON 98195

E-mail address: zhang@math.washington.edu 